\title{
XXIX.
}

\section{Beitrag zur Kenntniss der Kreislaufsverhältnisse der Säugetlierlunge.}

\author{
(Aus dem pathologisch-anatomischen Institute.)
}

Von Dr. Küttner in Heidelberg.

(Hierzu Taf. XI-XII.)

In einer früheren Mittheilung ${ }^{2}$ ) hatte ich den Nachweis zu führen gesucht, dass die Gefässbahnen der Froschlunge gewisse bis jetzt unbeachtete Verhältnisse darbieten und rechnete hiezu den Abgang engster Röhren aus relativ starken Arterien, die Weite des Capillarnetzes, so wie die relativ beträchtliche Stärke der Venen. Dabei wurde hervorgehoben, wie sich aus dieser Anordnung die grosse Geschwindigkeit, mit welcher das Blut die Froschlunge durchströmt, erklären lasse und in welcher Weise sich diese Eigenthümlichkeiten der Gefässbahn unter pathologischen Bedingungen geltend machen können. In dieser letzten Beziehung hatte sich ergeben, dass die Gapillaren die Function von Anastomosen zwischen $z$ wei Arterien und Arteriensystemen übernehmen k*önnen - dass sich waiter durch Ausschaltung der seitlichen Babuen kleinste, Arterienanastomosen vortäuschende Stämme in den Capillarnetzen ausbilden und dass drittens Venen; unter Zuhülf enahme von zwei capillären Bahnen, als Anastomosen zwischen zwei Arterien functioniren können.

Nachdem die. Beobachtung des Kreislaufs der Froschlungen solche für Physiologie und Pathologie gleich interessante Ergebnisse geliefert hatte, musste es meine nächste Aufgabe sein, zu untersuchen ob auch in der Säugethierlunge entsprechende Eigenthümlichkeiten der Gefässanordnung sich fänden, ob sich der einfache Lungensack des Frosches in dieser Beziehung mit dem Infundibulum

1) Beitrag zu den Kreislaufsverhältnissen der Froschlunge. Dieses Archiv Bd. 61, S. 21. 1874 . 

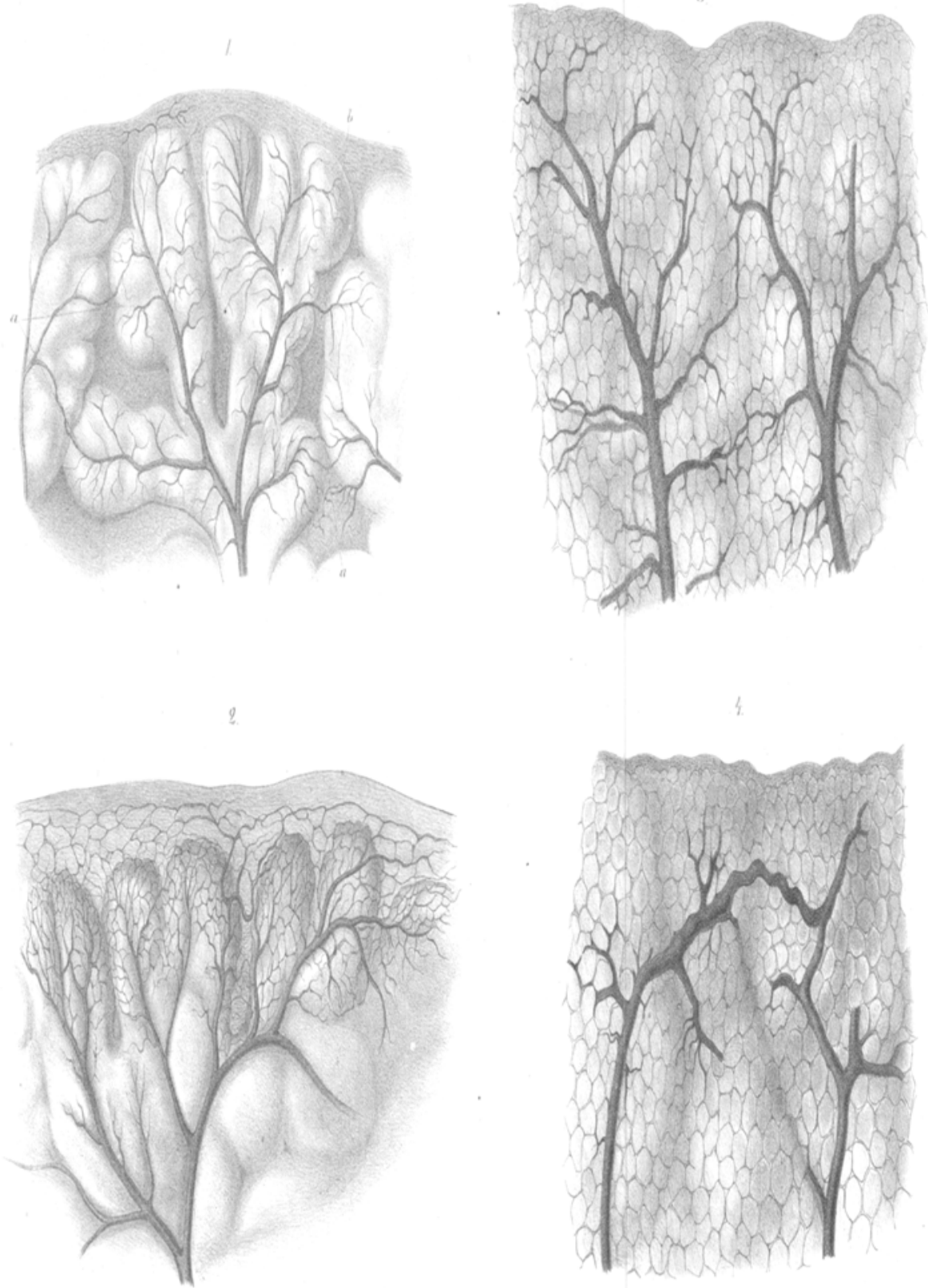

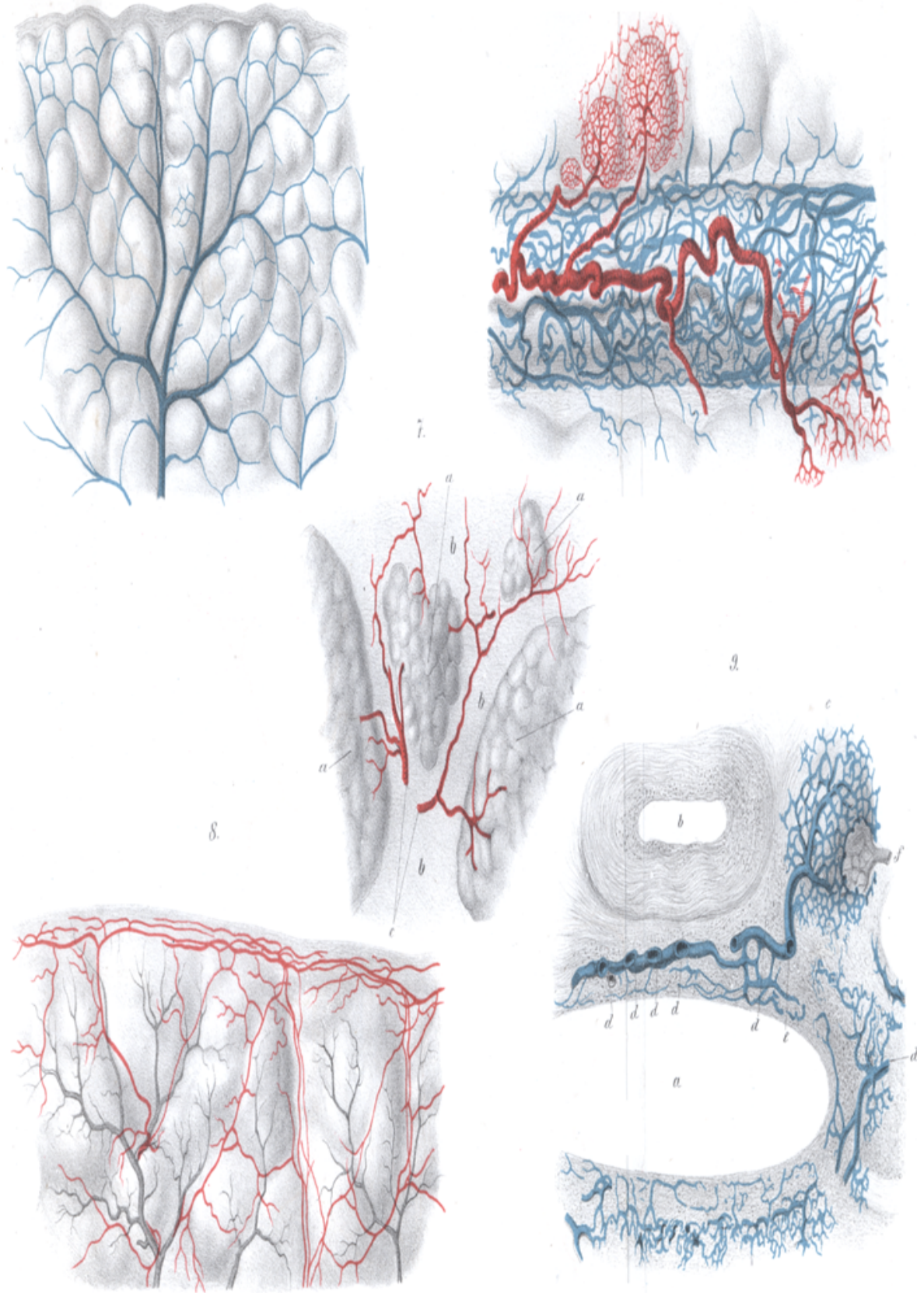
der Säugethierlunge vergleichen liesse und welche Besonderbeiten durch das Auftreten der Art. und Ven. bronchialis, eines Gefässsystems, das die Wandungen der Bronchien und das interlobuläre Bindegewebe mit Blut versorgt, bedingt seien.

Ich war bei meiner ersten Arbeit von eituer Untersuchung des todten Objects ausgegangen und hatte dan $n$ auf experimentellem Wege durch die Beobachtung an der Lunge des lebenden Frosches mir eine Einsicht in die Kreislaufsverhältnisse zu verschaffen gesucht. Dieselbe Methode habe ich auch bei dieser zweiten Arbeit befolgt; ich fing mit der Injection verschiedenartigster Massen bei getödteten Thieren an und ging darauf zu den Infusionen mit gelösten, insbesondere aber körnigen Substanzen beim lebenden Thiere über. Dem entsprechend folgt hier in erster Reihe die Mittheilung der Resultate der auf anatotomischem, in zweiter diejenigen der auf experimentellem Wege erlangten Ergebnisse.

\section{Anatomischer Theil.}

Wenn ich trotz der umfangreichen Literatur es unternehme über Lungengefïsse Mittheilungen zu machen, so bedarf es einiger Worte der Rechtfertigung. Es wollen mir solche um so mehr erforderlich dünken als Cohnheim und Litten ${ }^{1}$ ) den bedingten Werth der anatomischen Untersuchungsmethode für die Entscheidung der die normalen und pathologischen Kreislaufsverhältnisse betreffenden Fragen besonders betonen. Der dieser Methode gemachte Vorwurf müsste als berechtigt zugegeben werden, wenn man sich auf die Untersuchung von Schnittpräparaten injicirter Organe beschränken und aus solchen, ihres Gefässreichthums wegen vielfach unklaren Flächenbildern, auf die Blutvertheilung in den einzelnen Gefässbezirken der Lunge schliessen wollte. Beschränkt man sich nicht auf die Anwendung der einfachsten technischen Hülfsmittel, so lassen sich meiner Ansicht nach Thatsachen gewirnen, welche uns eine Einsicht in die complicirten Gefässanlagen der Lungen gestatten und für die experimentelle Bearbeitung des Gegenstandes eine sichere Basis schaffen.

Um diesen Anforderungen möglichst gerecht zu werden, habe ich die versehiedenartigsten Methoden in Anwendung gebracht. Was

') Cohnheim und Litten, Ueber die Folgen der Embolie der Lungenarterie. Dieses Archiv Bd. 65. 1875.

archiv f. pathol. Anat. Bd. IXXXII. Hft. 4. 
zunächst die Injectionsmethoden anbetriff, die hier in erster Reihe in Betracht kommen, so nahm ich zahlreiche Injectionen mit nicht diffundirenden in Wasser, Glycerin oder Leim suspendirten Stoffen vor. In einigen Fällen wurde eine möglichst vollständige Füllung der Gefässbahnen, in den anderen absichtlich eine nur theilweise ausgeführt. An Objecten erster Art ist das Bild oft ein sebr verworrenes, weil die Injectionsmasse nach allen Richtungen innerhalb der Gefässbahnen sich vertheilt, ganz gleichgültig von welehen Gefässstämmen aus die Injection vorgenommen wurde. Weniger vollständig injicirte Präparate zeigen dagegen in sehr typischer Weise die Verzweigung und die ganze Anordnung des eben injicirten Gefässbezirkes. Auch gleichzeitige Injectionen der einzelnen Gefässstämme mit verschiedenen Farbstoffen führte ich aus; sie lieferten aber die wenigst brauchbaren Objecte, weil die verschiedenen Farben in ganz unregelmässiger, willkürlicher Weise sich bald in diesem, bald in jenem Gefässbezirke verbreiteten, die Farbe des Gefässinhaltes keinen Schluss auf dessen Abstammung gestattete. Um mich über den Typus der Verzweigung der einzelnen Gefässsysteme zu unterrichten, fand ich es zweckmässig die capillaren Verbindungen zwischen denselben auszuschalten. Zu diesem Zwecke spritzte ich, etwa eine Viertelstunde vor der Injection der Gefässe mit farbigen Massen, in den Bronchialbaum des eben durch rasche und möglichst ausgiebige Verblutung getödteten Thieres, eine Silberlösung von $0,25-0,1 \mathrm{pCt}$. ein: es kommt auf diese Weise zu einer Gerinnung des Inhalts namentlich der Capillaren, weniger der Arterien und Venen; der später injicirte Farbstoff dringt deshalb nur bis zu den Capillaren vor, nicht aber in diese selbst ein. Wechselt man mit der Concentration der Silberlösung, die auch den Inhalt feinerer Arterien und Venen zu gerinnen. vermag, so lassen sich mit dieser Methode die Bahnen der einzelnen Gefässsysteme bis zu einer beliebigen Ausbreitung derselben für sich übersicbtlich demonstriren. Denn bei allen Gefässpräparaten handelt es sich in erster Linie darum, Arterien von Venen, eine Arteria pulmonalis von einer Art. bronchialis, eine Vena pulmonalis von einer Vena bronchialis und diese untereinander unterscheiden zu können, - es ist eine Vorbedingung zu jeder weiteren Untersuchung und Fragestellung, genau den Ort angeben zu können, wo Arterie und Vene liegt, sie gestattet überhaupt erst die Frage nach der pbysiologischen Bedeutung, 
die das eine oder das andere Gefäss im Lungenhaushalte zu vertreten hat, ob Anastomosen und zwischen welchen Gefässen sie namentlich vorkommen. Nur zusammenhängende, grosse Gefässbilder einzelner Systeme mit ausgeschalteten Capillaren können Aehnliches leisten, - die oben angegebene Vorbereitung des Materials glaube ich in dieser Hinsicht empfehlen zu können.

Zur isolirten Darstellung der einzelnen Gefässsysteme und Bezirke eignet sich auch die Ausspritzung derselben mit der von Hoyer ${ }^{1}$ ) empfohlenen Masse: sie besteht aus einer alkoholischen Lösung von Schellack und ist gefärbt mit in Salzsäure sich nicht zersetzenden körnigen Stoffen, welche die capillaren Bahnen zu passiren nicht im Stande sind; die mit ihr ausgegossenen objecte lassen sich sowohl corrodiren als auch zu Schnittpräparaten brauchen. Es ist eine Methode die im Wesentlichen Aehnliches leistet wie die von Cohnheim und Litten ${ }^{2}$ ) angewendete Aufschwemmung von chromsaurem Bleioxyd, nur dass letztere von genannten Autoren zu natïrlichen, zu Selbstinjectionen des lebenden Thieres gebraucht warde.

Was die Hyrtl'schen Corrosionsmethoden anbetrifit, so habe ich sie zur Darstellung der gröberen Gefässverzweigungen häufig angewendet; für die engeren Gefässe leisten sie weniger, - injicirt man in ein erwärmtes Object heisse Massen, so lassen sich diese wohl füllen, man erhält aber statt eines Gefässbaumes einen unentwirrbaren Gefässknäuel, aus dem sich hier und da wohl gute Bruchstücke auswählen lassen, die aber ibrer Undurchsichtigkeit und Zusammenhangslosigkeit wegen von untergeordnetem Werthe sind.

So weit die Methoden der Injection. Was das Material anbetrifft, welches injicirt werden soll, so ist die Wahl desselben nicht hoch genug anzuschlagen. Geht man von dem im Ganzen richtigen Satze aus, die Art. bronchialis sei das nutritive, die Art. pulm. das secretorische Gefäss der Lunge, so wịd er die Wahl in so fern beeinflussen als man das Material zur Injection a priori verwerthbar halten wird, in welchen Infundibula und Alveolen deutlich vom Bindegewebslager, von den grösseren Bindegewebszügen getrennt sind; mit anderen Worten, man wird Lungen wählen, die

1) Archiv für mikrosk. Anatom. Bd. 13. S. 604.

2) Dieses Archiv Bd. 65. S. 104. 
bindegewebsreich sind. In dieser Beziehung sind von Thierlungen die der Kaninchen am wenigsten zu empfehlen. Bessere Resultate erhielt ich bei Verwendung der Hundelungen, - als besonders brauchbares Material ergaben sich die Lungen älterer, namentlich menschlicher Embryonen, ebenso die Lungen Neugeborner. Injicirt man solche, und bringt sie, durch Eingiessen von ungefärbtem Leim oder Alkohol in den Bronchialbaum, auf ihre vitale Weite, so erhält man Bilder äber die Architectur und Gefässanordnung der Lungen wie sie kaum übersichtlicher gedacht oder gezeichnet werden können. Streng von einander geschieden findet man einerseits die zu Lappen und Läppchen combinírten Infundibula, den central in jedes Läppchen eintretenden Bronchus mit seiner epithelialen Auskleidung und seiner Verästelung, so wie die sie begleitenden und die Infundibula einspinnenden Gefässe, - andererseits das die Lobuli umgebende Bindegewebe mit dessen Nerven, Lymph- und Blutgefässen. Mit der Wahl dieses Materials wäre man zugleich dem Einwurf begegnet, dass bei Thier- und Menschenlungen wesentliche Verschiedenheiteu in dem Bau derselben vorhanden seien und dass an ersteren gemachle Wahrnehmungen nicht unbedingt auf letztere sich übertragen liessen. Es würde sich nur fragen, ob nicht Differenzen zwischen den Lungen Nengeborner und Erwachsener bestehen. Um diesem Einwande zu begegnen balse ich zahlreiche Lungen der letzteren injicirt und gefunden, dass Lungen von an katarrhalischen Pneumonien und an rasch verlaufender Perjbronchitis Verstorbenej, der serösen Schwellung des Bindegewebsgerustes wegen ein gutes Untersuchungmaterial geben und dass in der Gefässanordnung wesentliche Abweichungen nicht nachweisbar sind. Schmälert die Grösse des Organs auch dessen Uebersichtlichkeit, so sind die Präparate doch zur Controle des an embryonalen Lungen Gesehenen brauchbar.

Erwähnen will ich noch, dass ich den Bronchialbaum aller zu untersuchenden Lungen bald vor, bald nach der Injection der Gefässbahnen, mit Wasser, Leim oder Alkohol ausgoss, um sie zu ejner annähernd natürlichen Ausdehnung zu bringen, ohne welche selbst die bestgelungenen Injectionen wenig Werth haben. Nur auf diese Weise ist es möglich die terminalen Bronchien, ihre endständigen Infundibula und das sie begleitende Gefässnetz verfolgen zu können. Von den so vorbereiteten Lungen macbte jch aufein- 
anderfolgende Serien von Schnitten, um mir auf diese Weise eine Anschauung über die Anordnung der Gefässe nicht nur an einzelnen Infundibulis, sondern auch an Gruppen von solchen und in den zwischen ihnen gelegenen Bindegewebszïgen zu verschaffen. Besonders empfehlenswerth sind aber zum Studium dieser Verhältnisse die scharfen Ränder der einzelnen Lungenlappen; ich trage sie ab und lege sie ohne weitere Präparation unter das Mikroskop: da das Object an seinen beiden Flächen mit Pleura bedeckt ist, der scharfe Rand aus einer Reihe dicht neben einander liegender Infundibula besteht, so übersieht man, neben den Pleuragefässen in weiter Continuität den Verlauf der übrigen Gefässe auf und zwischen den Trichtern (Fig. 1, 2, 3, 4, 7).

Die in Anwendung gekommenen Methoden sind nicht nur zahlreich, sondern zum Theil auch neu oder wenigstens, behufs des Studiums der Gefässanordnung in den Lungen, noch nicht verwerthet, so dass man schon daraus eine Aufforderung zu deren Mitheilung ableiten könnte. Die eigentliche Rechtfertigung der nachfolgenden Erörterungen glaube ich aber darin erblicken zu dürfen, dass es mir mittelst dieser Methoden gelungen ist, eine Einsicht in die Gefässeinrichtungen der Lunge zu gewinnen, von denen einzelne noch keine Beleuchtung gefunden haben, während andere den Gegenstand einer noch heute offenen Discussion bilden.

Zu den ersteren zähle ich die Frage nach der Abzweigung der kleineren Gefässe von den grösseren, d. h. ob ähnlich wie in der Froschlunge, so auch in der Säugethierlunge kleine und kleinste Gefässe von verhältnissmässig grossen Stämmen entspringen. Dagegen wäre die Frage nach der gegenseitigen Beziehung der einzelnen Gefässbezirke, ob sonst Anastomosen zwischen denselben bestehen oder nicht und welcher Art diese sind den viel discutirten beizuzählen.

Die Wahrnehmung, dass bei der Injection des einen Gefässsystems die Masse in die anderen Gefässbezirke vordringt, ist wohl die Veranlassung gewesen, dass man eine ausgedehnte Beziehung zwischen den einzelnen Bezirken annahm. Als fraglich wurde es bezeichnet, ob diese nur durch Capillaren oder durch. weitere Gefässzweige vermittelt werde. So sagt z. B. Haller ${ }^{1}$ ) „cum ar-

1) Icones anatom. Götting. 17ă6. Tab. art. bronchialis. 
teria pulmonali evidentissimis et magnis anastomosibus broncbialis comunicat, - - Hae anastomoses fiunt tum a ramis ab arteria pulmonali bronchum conscendentibus, tum aliis a bronchiali de broncho descendentibus et in substantiam delatis ..." Hyrtl betont, dass ,schon Haller es gekannt babe, dass die Arteria bronchialis im Lungenparenchym kein abgeschlossenes, für sich bestehendes, nutritives Gefässsystem der Lunge bilde, sondern mit Verzwejgungen der Art. pulm. in anastomotischer Verbindung trete. Ich erhalte durch isolirte Injection der Art. bronch. das respiratorische Gefässnetz der Vesiculae ebenso gefüllt als wie bei Injection der Art. pulmonalis." An einer anderen Stelle sagt Hyrtl ${ }^{2}$ ) , bei Injection der Art. pulm. füllen sich auch die Capillargefässe der Bronchialschleimhaut". Weiter ${ }^{3}$ ) "die Capillargefässe aller Lobuli stehen in anastomotischer Verbindung". Einmal hebt er hervor, "dass kein einziges Corrosionspräparat Anastomosen zwischen Zweigen des Arterien- und Venenbaumes zeigt" ${ }^{4}$ ).

Henle ${ }^{5}$ ) giebt an, "da die Wand der Bronchien in der Wand der Alveolen sich ununterbrochen fortsetzt, so müssen an den Gienzen beider die Capillaren beider Gefässsysteme ineinander greifen ", "die Pulmonalarterie breitet sich auf der Bronchialschleimhaut aus".

Friedrich Arnold ${ }^{6}$ ): „Die feineren Zweige der Lungenschlagader treten zu den Bronchien und lösen sich in den Bronchien derselben in ein gröberes Capillarnetz auf."

Luschka ${ }^{7}$ ) giebt an, dass an gewissen Stellen ihrer capillaren Ausbreitung zwischen Art. pulm. und Art. bronchialis eine vielfache Anastomosirung obne Ausnahme stattfindet, "dass sich zahlreiche Zweige zur Wand der feineren Bronchien begeben, in deren Schleimbaut sich dieselben in ein Capillarnetz auflösen". "Während ihres Verlaufes giebt die Art. bronch. zahlreiche $\mathrm{Zweige} a b$, welche die äussere Faserhaut durchbohren und sich nunmehr in ein capillares Netz auflösen, das ununterbrochen in dasjenige übergeht, welches

1) Hyrtl's Anatomie S. 913. Aufl.

2) Hyrtl's Corrosionsanatomie. S. 97. 5.

3) Ebendas. S. 97. 7.

) Ebendas. S. 82.

5) Henle, Eingeweidelehre. 1866. S. 285.

6) Fr. Arnold, Anatomie. Bd. II. S. 171.

7) Luscbka, Anatom. des Menschen. Bd.I. 2. S. 314, 315, 317. 
daselbst durch die Erdzweige der Art. pulmon. erzeugt wird." "Zahlreiche Seitenzweige begeben sich in den serösen Ueberzug der Lunge, wo sie ebenfalls mit den Capillaren der Art. pulmon. anastomosiren."

Die angeführten Autoren sind also auf dem Wege der anatomischen Untersuchung zu dem Resultate gekommen, dass die Gefässgebiete der Art. bronch. und pulmon., sowie der Vena pulmon. keineswegs in sich abgeschlossen seien, sondern dass vielfache gegenseitige Beziehungen bestehen und zwar sollen diese nach der Ansicbt der Meisten durch capillare Gefässe vermittelt werden. Virchow hat zuerst auf experimentellem Wege dargethan, dass die Bronchialarterie für das nutritive Geschäft , der Lunge genüge und dass das functionelle Gefäss ohne Nachtheil für die Ernährung des Organs unterbrochen werden könne". Insbesondere aber wird von Virchow betont; dass ,mittelst des Experimentes die Herstellung eines collateralen Kreislaufes durch die Bronchial- und Intercostalarterien nach Verstopfung der Lungenarterie sich nachweisen lasse, zumal da die vielfachen Anastomosen $z$ wischen Lungenarterie und Bronchialarterje bestehen".

Im Widerspruche mit den eben berichteten Anschauungen stehen die Angaben Rindfleisch's, Cohnheim's und Litten's, insofern sie annehmen, dass die Gefässbezirke der Lungen mehr in sich abgeschlossene seien. So hebt Rindfleis $\mathrm{ch}^{2}$ ) hervor, ,dass nicht blos die allerkleinsten im Innern des Lobulus verlaufenden Aestchen isolirt sind, auch die zu einem Lobulus hinzutretenden, resp. zwischen zwei Lobuli verlaufenden Arterien einen vollkommen getrennten Verlauf haben". Er wendet den von Cohnheim in die anatomische Literatur eingeführten Begriff der Endarterie zuerst auf die Lungenarterie an und constatirt, dass "alle Hauptäste der Art. pulmon. Endarterien sind". Cohnheim und Litten ${ }^{3}$ ) stimmen dieser Auffassung bei und fügen hinzu: „es existire gar kein anatomischer Anhaltspunkt dafür, dass die Speisung der Gefässe eines Lungenbezirkes, dessen zuführende Lungenarterie verlegt ist, von den Zweigen der Bronchialarterie übernommen werde." Sie betoneu, "dass nach den Lehren der Anatomie, wie sie in den bekannten

1) Virchow, Gesammelte AbhandI. S. 295-296. Exper. XX. 12. Juni 1846.

2) Rindfleisch, Handbuch der pathol. Gewebelehre. 3. Aufl. S. 395.

$\left.{ }^{3}\right)$ Cohnbeim und Litten, Dieses Arch. Bd. 65. S. 102. 
Lehrbüehern der Anatomie vorgetragen werden, arterielle Anastomosen der Aeste der Pulmonalis unter einander ebenso wenig existiren sollen als solche $z$ wischen diesen und Zwejgen der Bronchialarterien - so wird z. B. auch in Hyrtl's Corrosionsanatomie der isolirte, anastomosenfreie Verlauf aller Zweige der Art. pulmon. ausdrücklich hervorgehoben".

Die von Haller, Fr. Arnold, Hyrtl, Henle, Luschka, Virchow u. A. zwischen den Verästelungen der Art. pulmon. und bronchialis angenommenen Beziehungen sind von Cohnheim und Litten nicht berücksichtigt worden, weil sie ausschliesslich auf experimentellem Wege die Frage zur Lösung zu bringen versuchten. Untersuchungen, die ich schon vor dem Erscheinen der Arbeit Cohnheim's and Litten's begonnen und zum grössten Theil zu Ende geführt hatte, ergaben mir ein anderes Resultat. Die Publication der letztgenannten Forscher ist die Veranlassung geworden, dass ich den Gegenstand noch einmal bearbeitete; in den nachfolgenden Zeilen ist in mögliehster Kürze das Resultat dieser mehrjährigen Untersuchung niedergelegt.

Ich beginne mit der Beschreibung der Art. pulm. und ihrer Verzweigungen.

\section{Die Arteria pulmonalis.}

„Die zur Lunge gehende Ader spaltet sich zunächst in zwei Zweige zu jeder Hälfte der Lunge hin; dann verläuft sie neben jeder Bronchialröhre und Oeffung immer in einer Breite, welche dem Durchmesser jener entspricht, so dass es keinen Theil der Lunge giebt, in welchem man nicht eine solche Oeffuung und einen Aderzweíg anträfe; die Adergänge liegen oberhalb der von der Luftröhre ausgehenden Röhren," - d. b. die Verästelungen der Arteria pulmonalis folgen unverrückt denen des Bronchialbaumes. Diese bereits von Aristoteles ${ }^{1}$ ) hervorgehobene wechselseitige Lagerung der Arteria pulmonalis und des Bronchus ist so constant, dass man in jedem Lungenpräparate das dem Bronchus zunächst liegende, mit ihm in einer Richtung ziehende, grössere Gefäss unfehlbar als Zwejg der Arteria pulmonalis wird ansprechen können. An embryonalen Lungen und Lungen Neugeborner sieht man beide

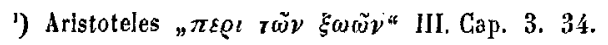


in einem Bindegewebslager liegen, das vom Lungenhilus anhebt, in den Hilus eines jeden Lüppchens mit ihnen eintritt und bier ausstrahlt. In diesen Läppchen verlaufen beide nicht nur dicht neben einander - es treten auch Aeste der Arteria pulmonalis zum Bronchus selbst und dringen bis zur Mucosa derselben, die übrigen Lagen des Bronchus der Arteria bronchialis überlassend. Mit dem Auftreten der terminalen Broncheolen ändert sich dieses Verhältniss: die Bronchialarterie hört als solche auf, die Pulmonalarterie oder besser deren Seitenzweige umspinnen die alveolaren Ausstülpungen, an ihren Aussenflächen ausschliesslich. An der Stelle, wo sich der terminale Bronchus in ein System endständiger Infundibeln spaltet - theilt sich der bisher verlaufende Stamm der Arteria pulm. in ebenso viel Aeste, d. h. pinselförmig; jedes Infundibulum bekomml sein Stämmchen, das sich federfahnenähnlich an seiner Aussenfläche ausbreitet (Fig. 1). Die Stämmchen verhalten sich zu ihren Aesten wie der Mittelnerv eines lanzettförmigen Blattes etwa zu den parallel zu einander gerichteten Seitennerven. Die terminalen Verzweigungen der Arteria pulmonalis umspinnen die terminalen Alveolen, d. h. die zu Infundibeln gruppirten Alveolencomplexe, die Einsenkungen, die Thäler zwischen sich den Venen überlassend (vergl. Fig. 1 u. Fig. 5). An gut ausgedehnten und injicirten Lungen lässt sich nicht nur das Lageverhältniss der Arteria pulmonalis zum Infundibulum and dessen einzelnen Alveolen übersehen, man erkennt auch den wechselseitigen Austausch von Arterien je zwei nebeneinander gelegener Infundibula und Lobuli oder mit anderen Worten das Gefässgebiet jedes einzelnen Stämmchens der Arteria pulmonalis hat mit dieser ihrer Ausbreitung in der Bronchialwand, auf den wandständigen Alveolen, den Alveolargängen und Infundibeln noch nicht ihr Ende erreicht. An jeder Lunge, sei sie eine embryonale, entwickelte oder pathologische, an der die Infundibula und Läppchen gut ausgedehnt sind, die ein reichliches Bindegewebslager aufauweisen haben, sieht man die Endverzweigungen der Arteria pulmonalis über die Grenzen der Infundibula und Lobuli hinaus in das interlobuläre und subpleurale Bindegewebe treten und sich hier entweder capillär auflösen oder zur Peripherie eines benachbarten Acinus treten, in den Capillaren dieses sich verlierend. Sie sind in sofern von Interesse, als sie die Bezirke verschiedener Pulmonalarterien unter einander verbinden oder speisen, wenn das 
sich auschliessende Capillarnetz spärlich ist oder dessen Gefässe verlegt sind. Diese Bilder lassen sich leicht erreichen, - wird der Bronchialbaum mit einer entsprechend starken Silberlösung ausgegossen und erst später die Arteria pulmonalis mit einer farbigen Masse injicirt, so verbreitet sich letztere vorwiegend in den grösseren, intacten Zweigen, - nur wenige der Capillaren bleiben hier und da zufällig intact, d. h. ihr Inhalt gerinnt nicht, sie werden weiter und bringen auf diese Weise die Verbindung verschiedener Arterienbezirke zu Stande (Fig. 3,4 ).

Das wäre im Grossen der Verlauf des Stammes der Arteria pulmonalis vom Hilus der Lunge bis zu deren Peripherie, beziehungsweise der Peripherie eines jeden Lungenläppchens. Ich habe das stete Beisammenbleiben von Bronchus und Arteria pulmonalis namentlich hervorgehoben, weil ich der Ueberzeugung bin, dass die Kenntniss der Lage beider wesentlich zum Verständniss eines beliebigen Lungenpräparates beiträgt. Aus diesem steten Beisammenbleiben von Arteria pulmonalis und Bronchus resultirt eine Eigenthümlichkeit der ersteren: während ihrer Entwickelung lehnt sie sich dem Brouchialbaume an; dieser wächst an seinem Scheitel ungetheilt, monopodisch fort, wäbrend seitliche Sprossen am Stamm hervortreten; wie sich dieser nicht dichotomisch verzweigt, ebenso wenig thut es die Arteria pulmonalis, - von einem stärkeren Stamme zweigen sich relativ feine Seitenzweige ab. An einem völlig entwickelten Bronchialbaum lässt sich dessen früheres monopodisches Wachsen schwer wiedererkennen, - die Art der Injection lässt, sowobl am Bronchus als auch an der Arteria pulmonalis, die Monopodie häufig zurü $\mathrm{ek}-$, das Princip der dichotomischen Verzweigung aber vortreten. Am deutlichsten erkennt man erstere am linken, unteren Lungenlappen jeder Säugethierlunge; bei einer bestimmten Schnittrichtung gelingt es beide von ihrem Ursprunge am Hilus bis zu ihrer endlichen Ausbreitung im scharfen Lungenrande, also in ihrem ganzen Verlaufe, als mächtige H́auptstämme blosszulegen, von denen relativ enge Seitenzweige rechtwinklig abgehen. Ich glaube diese Verzweigung der Arteria pulmonalis als eine Einrichtung constatiren zu müssen, die wesentlich zur Strombeschleunigung in derselben beiträgt.

Was die Ramification sonst anbetriff, so zweigen sich rom Hauptstamme der Arteria pulmonalis die Nebenstämme mehr oder 
weniger rechtwinklig $a b$, in den Läppchen ist die Insertion eine mehr spitzwinklige; es lässt sich weiter constatiren, dass Gefässe von gleicher Stärke in einer annähernden Spirallinie aus dem jedesmaligen Mutterstamm treten, ein Verhältniss, auf das Hyr $\mathrm{t}^{1}$ ) hingewiesen hat (Manatus australis).

Habe jch bis jetzt Thatsachen angefuhrt, die. sowohl der Arteria pulmonalis als auch den übrigen Körperarterien mehr oder weniger gemeinschaftlich sind, so gehe ich nunmebr zu den Eigenthümlichkeiten der ersteren über. Hierzu rechne ich den Abgang feinster Gefässe aus relativ starken Stämmen. Sie sind bereits an Injectionspräparaten deutlich, die mit wässerigen, farbigen Massen ausgeführt wurden - werden aber nm vieles klarer, so man sich der Corrosionsmassen bedient. Ist die Masse eine recht zähe, die Corrosion vorsichtig ausgeführt, so erkennt man an jeder Arteria pulmonalis bereits mit unbewaffnetem Alige die uberaus feinen Arterienwurzeln als dicht neben einander stehende Härchen und Borsten, die der Oberfläcbe des Stammrohres ein eigenthümliches, pelziges Gepräge geben. Mikrometrisch bestimmt würde sich nach vielen Messungen, die ich gemacbt, etwa Folgendes sagen lassen: von einem Gefäss von $0,136 \mathrm{Mm}$. zweigten sich dicht neben einander und der Reihe nach Arterienwurzeln ab von $0,033 \mathrm{Mm}$., $0,016 \mathrm{Mm} ., 0,011 \mathrm{Mm} ., 0,010 \mathrm{Mm}$.; von einem anderen Stammgefäss von $0,619 \mathrm{Mm}$. entwickelten sich ebenfalls Reiserchen von 0,017 0,08-0,04-0,01 Mm. Berücksichtigt man nun, dass in demselben Corrosionspräparate die Durchschnittsweite der Capillaren sich mit $0,009 \mathrm{Mm}$. bestimmen liess, so ergiebt es sich, dass von relativ mächtigen Arterien der Stärke nach nahezu capillare Arterienwurzeln als Seitenäste sich abzweigten.

Was das Gefässgebiet dieser Arterienwurzeln anbetriff, so verhalten sie sich je nach ihrer Stärke verschieden: die feinsten lösen sich sofort als Vasa vasorum capillär auf, - die stärkeren gelangen bis in das perivasculäre oder peribronchiale Bindegewebe und verästeln sich hier capillär, - oder aber sie treten zur Oberfläche der zunächst liegenden Läppchen und verschwinden mit ihrer Auflösung in den Capillarbahnen der Alveolen. Interessant ist bun die Thalsache, dass die Endverzweigungen einer und derselben

1) Hyrtl, Corrosionsanatomie. S. 82. 
Stammarterie sich verschieden verhalten, je nachdem sie sich im Bindegewebe oder an den Alveolen auflösen: im ersten Falle bilden sie weite Maschen und enge Röhren und unterscheiden sich in Nichts von der capillaren Auflösung der übrigen Körperarterien, im anderen Falle sind die Maschen eng, die Gefässe aber, wie alle Capillaren der Lungenalveolen, weit.

Um bei den capillären Auflösungèn der Arteria pulmonalis zu bleiben, habe ich einer ferneren Eigenthümlichkeit derselben zu gedenken. Verfolgt man zwei beliebige, dicht neben einander aus einem Stamm entspringende Arterienwurzeln bis in ihre capilläre Auflösung, sei diese nun im Bindegewebe oder in den Alveolen, so bemerkt man, dass dieselben mit noch anderen in ein gemeinschaftliches Capillarnetz übergehen, dessen venöse Abflüsse in keiner Weise mit den arteriellen Bahnen parallel laufen, sondern ihre besonderen Wege einseblagen ${ }^{1}$ ). So erschejnen die Capillarbahnen als ein zusammenhängendes, die ganze Lunge durchaiehendes Netz. Es ist dieses Princip der Verästelung so durchgehend an der Arteria pulmonalis entwickelt, dass es nicht nur an engen, dicht bei einander entspringenden Arterienwurzeln, sondern an starken Stämmen und deren endlichen, gegeneinander laufenden Verästelungen deutlich zu übersehen ist. Klar werden diese gemeinschaftlichen Capillarnetze verschiedener Stämme der Arteria pulmonalis, wenn man, wie jch bereits früher erwähnt, vor der Arterieninjection den Bronchialbaum mit einer $0,5-0,1$ procentigen Silberlösung ausspritzt, d. h. die mehr seitlichen Capillaren auf diese Weise ausschaltet. Es versteht sich hier von selbst, dass das Versuchsthier durch reichliche Verblutung rasch getödtet, dass die Lunge weit ausgedehnt sei, damit der gestreckte Verlauf der Gefässe übersichtlich werde; anzuempfehlen sind die intacten, scharfen Lungenränder. An diesen Präparaten lassen sich die Gefässe von ihrem Ursprunge bis zu ihrer endlichen Auflösung verfolgen, - an ihnen kann man sich davon uberzeugen, dass ein bedeutender Theil der Arteria pulmonalis sich im interlobulären Bindegewebe auflöse, d. h., dass sie nicht ausschliesslich eiu secretorisches Gefäss sej, - dass „die Capillarnetze aller Lobuli in anastomotischer Verbindung stehen" ${ }^{2}$ ).

5) Gohnheim und Litten, Dieses Areh. Bd.65. S. 109.

2) Hyrtl, Corrosionsanatomie. 95. 7. 
Meine anatomischen Untersuchungen haben mich weiter zu dem Ergebniss geführt, dass zwischen den Zweigen der Arteria pulmonalis keine Anastomosen vorkommen; trotz reichlichen Injectionsmaterials ist es mir niemals gelungen, Verbindungen anzutreffen, die ich anstandslos für arterielle Anastomosen hätte halten können. Ich habe aber constatiren müssen, dass unter bestimmten Bedingungen Verbindungen zwischen grösseren Zweigen der Arteria pulmonalis zu Stande kommen. Die früher mitgetheilten Beobachtungen an Froscblungen ${ }^{1}$ ), an denen ich unter meinen Augen, bei künstlich eingeleiteten Druckdifferenzen, capilläre Gefässe zu weiten, grössere Gefässzweige verbindenden Röhren sich umgestalten sah, bestimmen mich zu der Annahme, dass unter gegebenen Verhältnissen auch in der Säugethierlunge mächtigere Verbindungen zwischen grösseren Pulmonalarterienzweigen durch Erweiterung capillärer Gefässe entstehen können und das um so mehr, als der Typus der Arterienverästelung in beiden derselbe ist. Wesentlich begünstigt wird dieses Zustandekommen künstlicher Anastomosen durch eine Eigenthümlichkeit in der endlichen Verästelung. Ich habe eben erwăhnt, dass die unmittelbar aus Arterien stammenden Capillaren sich nicht in jedem Falle unmittelbar zu Venenstämmchen sammeln, sondera ihrerseits mit Arterien zusammenhängen; verfolgt man nun zwei, in gestrecktem Winkel gegen einander laufende Stämınıchen der Arteria pulmonalis, so überzeugt man sich davon, wie überaus nabe die beiderseitigen arteriellen Endäste an einander treten, ohne zu anastomosiren, - man üherzeugt sich weiter davon, dass die Capillaren nur eines oder $\mathrm{zw}$ wier Alveolen sie trennen. Diese ungemein kurzen, zwischen zwei Arterien eingefügten Capillaren sind es, die bej Druckdifferenzen in der einen oder der anderen Arterie, ähnlich wie in der Froschlunge, weite Verbindungen werden zu Wege bringen müssen. Fast ausnahmslos gelingt es, sogar bei Injectionen mit Hoyer'scher Corrosionsmasse, diese überaus kurzen Capillarstrecken mit zu füllen und Verbindungen zweier Arterien herzustellen, die um so deutlicher hervortreten, als alle übrigen Capillaren ausgeschaltet, verlegt sind. Auch hier muss ich hervorheben, dass eine wesentliche Bedingung, um diese künstlichen Anastomosen zu sehen, in einer entsprechenden Ausdehnung der Alveolen resp. der ganzen Lunge bestebt.

') Dieses Arch.' Bd. 61. 
Ich stimme somit denjenigen Forschern bei, die annehmen, dass zwischen den grösseren Zweigen der Arteria pulmonalis keine weiteren Anastomosen präformirt vorkommen. In diesem Sinne könnte man also die Pulmonalarterie als "Endarterie " bezeichnen. Auf der anderen Seite aber darf man nicht vergessen, dass solche Verbindungen jeden Augenblick entstehen können und die Arteria pulmonalis dann den Typus einer sogenannten ,Endarterie“" einbüsst; wenigstens werden dann die Strömungsverhältnisse in ihr dieselben sein können, wie in einem Gefäss, das nicht nach dem Typus der Endarterie angelegt ist. Ausserdem muss ich betonen, dass das Gefässgebiet der Arteria pulmonalis kein so abgeschlossenes ist, wie dieses Cobnheim und Litten annehmen, dass vielmehr Zweige derselben von einem Lobulus zu einem benachbarten übersetzen, dass andere in dem subpleuralen und interlobulären Bindegewebe und in der Bronchialwand sich verbreiten.

Bevor ich mich den übrigen Gefässsystemen der Lunge zuwende, muss ich in aller Kürze eben dieses Verhältnisses der Arteria pulmonalis zur Arteria bronchialis und zum Bronchus gedenken. Ich habe bereits erwähnt, dass an den Grenzen der capillaren Bronchien und Infundibulargänge die Capillarbezirke beider unmittelbar in einander übergehen, dass die Bronchialarterie als solche aufbört; weitere Beziehungen zwischen beiden Gefässgebieten lassen sich durch nachfolgende Thatsachen beweisen. Injicirt man die Lunge eines eben verbluteten Thieres von der Arteria pulmonalis aus, so tritt bereits mit dem Begins der Injection grossblasiger Schaum aus der Trachea. Es dauert nicht lange, so wird er gefärbt, kurze Zeit nachher fliesst die Injectionsmasse reichlich aus der Trachea und will man sonst eine gute Füllung der Arteria pulmonalis erreichen, man gut thun wird, die Trachea zu unterbinden. Bei der mikroskopischen Untersuchung soleher Objecte findet man eine vollständige Füllung der Gefässe der Bronchialwand bis in die subepitheliale Schicht hinein: eine Thatsache, die um so interessanter ist als, wie später ausgeführt werden soll, eine annähernd ähnliche Injection dieser Gefässbahnen bei Füllung von der Arteria bronchialis aus, wenigstens bei einzelnen Thieren, also namentlich beim Kaninchen sich kaum erreichen lässt.

Injicirt man weiler dic Arteriae bronchiales nach unterbundener Aorta ascendens und Carotiden von der Aorta thoracica oder abdo- 
minalis aus, so fliesst die Injectionsmasse nicht zur Trachea heraus, wohl aber erhält man eine Injection der Lunge. Ein genaueres Eingehen in die mikroskopischen Bilder ähnlich injicirter Lungen, verspare ich mir auf das Capitel, wo jch uber die Bronchialarterien speciell werde handeln müssen; an dieser Stelle sei nur bemerkt, dass sich Wechselbeziehungen der Arteria bronchialis und der Arteria pulmonalis constatiren lassen.

\section{Die Vena pulmonalis.}

Nur am Hilus der Lunge liegen Bronchus, Arteria und Vena pulmonalis dicht nebeneinander. Im weiteren Verlaufe weichen sie auseinander und zwar der Art, dass die Arteria und der Bronchus stets beisanmen bleiben, während die Vena ihre eigene Bahn verfolgt. So willkürlich diese auf den ersten Blick zu sein scheint, so schwer sie sich au der ausgewachsenen, geathmethabenden Lunge übersehen lässt, so einfach gestaltet sich der Verlauf der Vene an embryonalen Lungen. Die Zweige derselben sind vom Hilus bis zu ihrer capillären Auflösung in den interlobulären Bindegewebszägen gelegen. Sie bilden an den äussersten Grenzen der Läppchen weite Blutbuchten, in welche sich die Venen der Infundibula mit kurzen Stämmchen einsetzen. An den Trichtern selbst sind es die einspringenden scharfen Ränder der interalveolären Septa, welche die Capillaren aufnehmen. Der Einblick auch in diese Verhältnisse wird ausserordentlich klar, wenn man nach der früher angegebenen Methode durch Silberinjection die Capillaren, mit ihnen das ganze arterielle System, ausschaltet und dann erst die Venen injicirt. Vergleicht man solche Objecte mit Präparaten, an denen die Arterien ausschliesslich injicirt sind, so erkennt man an den letzteren, dem Centrum je eines Lobulus entsprechend, die dicht neben dem Bronchus verlaufende Pulmonalarterie, an den ersteren die Venen peripher gelagert - oder mit anderen Worten, die Arterie liegt intralobulär, die Vene interlobulär. Das Erkennen und Auffinden der Vena pulmonalis an Lungen Erwachsener ist schwieriger; in den ersten Lebensjahren schwinden die bedeutenden Bindegewebslager und man erkennt die Venen als im Durchmesser, weite, diinnwandige, von Alveolen allseitig umschlossene Gefässe oder Gefässlumina, - sie sind im Lungenparenchym in gewissen Abständen wie eingesprengt und es feblt der Bronchus in ihrer unmittelbaren 
Nähe. Bei der Untersuchung scharfer Lungenränder kann man sich davon überzeugen, dass die Verzweigungen der Venen in den Thaleinsenkungen zwischen den Infundibula und Alveolen verlaufen (Fig. 5).

Corrosionspräparate der Venen, mit denen der Arterien verglichen, geben charakteristische Unterschiede; an den letzteren ist diẻ Verzweigung der grösseren Stämme eine melır rechtwinklige, baumartige, an den ersteren eine spitzwinklige, es sind breite, gradlinig verlaufende Sammelstämme, an denen sich kurze Aestchen inseriren.

Da ich über die Beziehung der Gefässe der Bronchialwand zu der Pulmonalvene später zu berichten Gelegenheit haben werde, beschränke ich mich hier mit dem Hinweis auf die Thatsache, dass ein grosser Theil rom Bronchus kommender Venen in die Vena pulmonalis sich einsenken (Fig, 9).

Bezüglich des Verbaltens der Pulmonalvene wäre hervorzuheben, dass die $Z$ weige derselben ausnabmslos in den interlobulären Bindegewebszügen gelegen sind und daselbst grosse Sammelplexus bilden, in welche relativ feine, kurze Gefäss e sich einsenken.

\section{Gefässe des Bronchus.}

Es ist bereits darauf hingewiesen worden, dass bei einer Injection der Lungen von der Arteria pulmonalis aus die Gefässe der Bronchialwand, namentlich die der Mucosa sich vollständig füllen lassen, - dass die Füllung derselben ungleich schwächer wird bei Injection der Arteria bronchialis oder Aorta, - dass sich bei letzterer aber eine Füllung des Lungenparenchyms erreichen lässt. Diese Thatsachen drängen zu der Annahme, dass ausser den aus der Aorta entspringenden Gefässen auch Zweige der Arteria pulmonalis in die Bronchialwand sich einsenken, weiter, dass ausser der Arteria pulmonalis Zweige der Arteria bronchialis des Lungenparenchyms mit Blut versorgen. Eine weitere diesen Thatsachen entsprechende Eigenthümlichkeit findet sich aber auch in der Anordnung der Venen in der Bronchialwand, - diese stehen nicht nur mit Zweigen der Azygos und Cava superior, sondern auch mit denen der Palmonalvene in Verbindung. Ich unterscheide deshalb folgende Arten von Bronchialgefässen: 
a. Zweige, die aus der Aorta

b. Zweige, die aus der Pulmonalarterie stammen,

c. Zweige, die zur Vena azygos und Cava superior

d. Zwejge, die zur Pulmonalvene gehen.

a. Zweige, die aus der Aorta stammen.

Beim Menschen giebt es wenig Arterien mit so wechselndem und verschiedenem Ursprunge wie die Arteriae bronchiales. Unter 24 namentlichen Aufzeichnungen über diesen Gegenstand, welche Halle ${ }^{1}$ ) angestellt hat, ergab sich nur in 12 Fällen das gleiche Verhaiten; in den 12 anderen war der Ursprung ein in jedem Falle wechselnder. In den 12 annähernd ähnlichen Fällen entsprang die rechte Bronchialis posterior und die linke obere aus einem Truncus communis, welcher auch die rechte Intercostalis suprema abgab; die linke untere Bronchialis posterior stammte aus der Aorta descendens oder der 3.--4. Intercostalis sinistra. Krause ${ }^{2}$ ) giebt unter den Varietäten des Aortensystems $Q u$ ain' ${ }^{3}{ }^{3}$ ) Angaben ïber den Ursprung der Arteriae bronchiales an (dort nachzulesen). Im Allgemeinen lässt sich sagen, dass 2 Arteriae bronchiales posteriores von der Aorta descendens zur hinteren Wand der beiden Luftröhrenäste treten und diese durch das Lungenparenchym begleiten. Da die Aorta auf der linken Seite liegt, so entsteht die Arteria bronchialis dextra häufig nicht aus ihr, sondern aus der Intercostalis suprema dextra, die ihrerseits wieder verschiedene Ursprungsstellen hat (Subclavia). Die Bronchiales anteriores entstehen aus der Arteria mamaria interna. Von allen diesen Arterien lässt es sich behaupten, sie verfolgen mit ihren Hauptstämmen den Bronchus auf seinen Wegen durch das Lungenparenchym und geben unbedeutende Seitenzweige in das Bindegewebslager ab. Aber es ist noch einer letzten Art von Arterien zu gedenken, die sich selbstständig im Lungenbindegewebe verzweigen, ohne die Bronchialwand zu berühren; sie stammen aus den Arteriae oesophageae, mediastinales, pericardiales, verästeln sich in der Pleura mediastinalis, treten mit dieser zum Lungenhilus und bilden theils ein zusammenhängendes Netz von Pleuraarterien, theils breiten sie sich im inter-

1) Haller, Icones anatom. Fasc. III. p. 35-37.

2) Krause in Henle's Handbuch der Gefässlehre des Menschen. S. 203.

3) Quain. (184a) Taf. XXIV. 5.

Archivf.pathol. Anat. Bd. LXXIII. Hft. 4 . 
lobulären Bindegewebe aus. $O b$ man diese mit Recht $z u$ den Bronchialarterien zu zählen hat, lasse ich dahingestellt sein, jedenfalls führen sie der Lunge Aortenblut $\mathrm{zu}$, - ich habe sie hier angeführt, theils weil ihrer wenig gedacht wird, theils weil bei bestimmten Thierspecies sie es sind, die, deutlicher entwickelt, functionell in den Vordergrund treten (Kaninchen).

Bei dem Hunde nimmt die Arteria bronchialis posterior, einen kurzen gemeinschaftlichen Stamm mil der 6. rechten Intercostalis bildend, etwa in der Höhe des 6. Brustwirbels aus der Aorta thoracica ihren Ursprung, spaltet sich in eine rechte und linke, von denen die erstere noch eine Arteria oesophagea abgiebt; der Abgang einer Arteria bronchialis posterior aus der Intercostalis suprema ist, meinen Untersuchungen nach, der seltenere Fall. Was die Arteriae bronchiales anteriores anbetrift, so stammen sie theils aus der Arteria mamaria interna, theils aus der Trachealis descendens; kleinere Aeste datiren aus den Arteriae pericardiales und senken sich mit den Mediastinales und den Tracheooesophageae in den Lungenhilus ein.

Nicht unerwähnt möchte ich hier lassen, dass es mir bei Menschen- und Hundelungen hier und da wohl geglückt ist, die Bronchialmucosa von der Aorta aus vollständig zu injiciren.

Was das Verhalten der Arteria bronchialis beim Kaninchen anbelangt, so ist zu erwähnen, dass aus den ziemlich symmetrisch auf beiden Seiten längs den Köpfen der ersten bis dritten Rippe nach abwärts steigenden Arteriae intercostalis primae neben Art. mamariae, pericardiales und oesophagea auch eine Bronchialis sich entwickelt ${ }^{1}$ ). Dieselbe ist aber selbst bei sehr grossen Bastardthieren von so feinem Caliber, dass sie sich nur mit Mühe bis zum Lungenhilus verfolgen lässt. Ausserdem senken sich Zweige der Arteria trachealis und tracheooesophagea, welche aus der anonyma entspringen, in den Lungenhilus ein; allein auch sie sind äusserst fein. Ueberhaupt muss ich betonen, dass die Kaninchenlungen für die Untersuchung der in Rede stehenden Verbältnisse die wenigst brauchbaren Objecte sind. Bei vielen von der Aorta aus gemachten Injectionen habe ich keine oder doch nur äusserst unvollständige Füllungen der Gefässe der Bronchialwand erhalten. Es erklärt sich dies wohl aus dem Umstande, dass sowohl die Bronchialarterien

") Krause, Anatomie des Kaninchens. S. 188. 
stricte sic dictae, als auch die übrigen zum Pulmonalhilus tretenden $\mathrm{Z}$ weige der Mediastinalis, Pericardialis, Intercostalis etc. etc. ihres geringen Durchmessers wegen durch gerinnendes Blut leicht unwegsam werden. Man hat um so mehr Grund, diese Gerinnungen als maassgebend für das Nichtzustandekommen von $k$ ünstlich en Injectionen anzunehmen, als Infusionen mit bestimmten Färbemitteln am lebenden Thier vollständige Füllungen äusserst feiner, zum Lungenhilus tretender Arterien zu Stande bringen, diese aber ein eingehendes Studium der fraglichen Gefässanordnungen ermöglichen; es lässt sich constatiren, dass dem Lungenhilus von allen Gefässen der serösen Häute des Mittelfellraumes auf vielen Wegen Blut zufliessen könne, - die äusserst feinen Bronchialarterien scheinen auf diese Weise im Stande za sein, Störungen zu compensiren. Ich habe dieser Verhältnisse ausführlicher gedacht, weil ohne Kenntniss derselben eine richtige Beurtheilung der später mitzutheilenden Versuchsresultate unmöglich ist.

Was die aus der Aorta stammenden Zweige der Bronchialwand anbetrifft, so lässt sich im Allgemeinen sagen, dass ein Theil derselben direct aus der Aorta, ein anderer aus den Intercostales und Mamariae internae tritt; die zum interlobulären und subpleuralen Bindegewebe tretenden Arterien aber ihren Ursprung aus den Oesophageae, Mediastinales, Pericardiales nehmen.

Ueber die Verzweigung der Bronchialarterien am Bronchus, d. h. innerhalb der Lunge, macht Haller ${ }^{1}$ ) folgende Angabe: „Caeterum arteria bronchialis sive dextra sive sinistra, in pulmonem cum broncho demersa, in tot ramos finditur quot ipsius sunt bronehi, qui valde flexuoso ducto eos ramos sequuntur, non singuli sed in uno ramo bini ternive" (Fig. 6). Diese Arterienknäule oder spiraligen Umdrehungen sind sehr häufig, bereits an embryonalen Lungen vorhanden und so charakteristisch, dass sie jedes in dieser Weise gewundene Gefäss als Bronchialarterie kennzeichnen. Dabei ist der Verlauf der Arterien ein sebr in die Länge gestreckter; sie giebt im Vergleich mit den anderen Lungengefässen nur spärliche Seitenzweige $a b$, behält selbst in grossen Entfernungen einen nur allmählich abnehmenden Durchmesser. Bis in ihre feinsten Verästelungen zeichnet sie sich durch eine starke Ringfaserhaut aus and bekundet hiermit ihren Ursprung aus der Aorta.

1) Icones anatomicae. Gotting. 1754. Tabul. art. bronch. 
Das Geschick der Zweige der Bronchialarterie ist ein verschiedenes; die Einen verlaufen an der äusseren Fläche der Knorpelringe in der äusseren Faserhaut und schicken feinste Seitenzweige durch diese und die Muskelhaut zur Mucosa - deutlich lassen sie sich hier in dem Gewirre anderer Gefässe, von denen ich später zu reden haben werde, ihrer Ringfaserhaut wegen erkennen. Die Anderen treten zu den peribronchialen und perivasculären Bindegewebsscbejden; auf diesen Wegen umspinnen sie die Adventitia der dem Bronchus zunäcbst liegenden Arteria pulmonalis, die Nerven und Lymphgefässe, die beiden letzteren häufig mit so engem Netze, dass es mir vorgekommen ist, das Kittleistennetz des Lymphgefässendothels gefärbt zu erhalten. Am interessantesten sind die $\mathrm{Z}$ weige, die direct aus den Stämmen der Arteria broncbialis entspringen, das peribronchiale Bindegewebe durchbrechen, an benachbarte Infundibula treten und sich hier capillär auflösen (Fig. 6). Ich habe bereits erwähnt, dass an den Grenzen zwischen den kleinsten terminalen Bronchien und Alveolargängen die Arteriae bronchiales als solche aufhören und dass die Capillargebiete derselben unmittelbar in die der Art. pulmonalis übergehen. Während also im Allgemeinen die Regel gilt, dass die Verzweigungen der Arteriae bronchíales bis zu den Alveolargängen sich erstrecken, kommt eine Speisung der Infundibula mit Aortenblut in der Weise zu Stande, dass von relativ mächtigen Stämmen der Bronchialarterien Aeste unter nahezu rechtem Winkel abgehen und sicb an benachbarten Infundibeln capillär verzweigen. Die so gespeisten Capillaren unterscheiden sich, was die Weite ihres Rohres und die Weite ibrer Maschen anbetrifft, in Nichts von den übrigen Capillaren der Alveolen, die ihr Blut aus der Arteria pulmonalis nebmen. Die Bilder, die man namentlich bei embryonalen und Lungen (menschlicher) Neugeborner erhält, sind so klar, dass sich die Thatsache constatiren lässt, ausser der Arteria pulmonalis versorgt die Arteria bronchialis die Infundibula und Alveoli mit Blut.

Es bleiben mir noch die Aortenzweige zu besprechen übrig, die aus den Arteriae pericardiales, mediastinales, oesophageae stammen, mil der Pleura mediastinalis zum Lungenhilus treten und sich hier, in einer gewissen Selbständigkeit von den Arteriae bronchiales, im Bindegewebe verbreiten.

Man eröffne durch Resection eines entsprechend grossen Rippen- 
stückes die Brusthöhle eines ausgiebig verbluteten Thieres (Hund), spalte vorsichtig die Pleuraumhüllung an der Lungenwurzel und löse sie vom Bronchus. Nun unterbinde oder durchschneide man letzteren in der Weise, dass vorausgesetzt werden kann, man habe die grossen, aus der Arteria intercostalis und Mamaria interna entspringenden Bronchialarterien mit unterbunden oder durchschnitten, - die Gefässe der pleura mediastinalis aber geschont. Injicirt man nun die Lunge von der Aorta abdominalis aus, so ergiebt die Untersuchung bereits mit blossem Auge eine Füllung des Lungenparenchyms. Die mikroskopische Untersuchung parallel zur Oberfläche der Lunge geführter Schnitte, also Pleuraschnitte, giebt eine zusammenhängende Injection der Pleuraarterien, die ein völliges Gefässnetz im subpleuralen Bindegewebe bilden, an die Oberfläche der einzelnen Acini herantreten und sich an deren Trichter und Alveolen capillär ausbreiten. Die grösseren Stämmchen erinnern bis 'auf die spiraligen Knäule, welche fehlen, an die Arterien der Bronchialwand (Fig. 7). Untersucht man die beiderseits von Pleura überzogenen scharfen Lungenränder in toto, so sieht man, wie die eben erwähnten Pleuraarterien, bevor sie zur Lungenoberfläche kommen, Zweige in die interacinösen Bindegewebszïge schicken. Auf ihrem Wege geben sie Gefässreiser ab, die zu den Trichtern treten und sich hier capillär auflösen. Trotz des unterbundenen oder durchschnittenen Bronchus resp. Bronchialarterien sind die Arterien der Bronchialwand injicirt. - Schliesslich und das wäre mit das Interessanteste, erkennt man Anastomosen zwischen den Pleuraarterien und den intraacinösen Stämmchen der Arteria pulmonalis: die Füllung der letzteren ist miltelst der Pleuraarterien von der Aorta aus zu Stande gekommen (Fig. 8).

Da die weitere Beschreibung eine complicirte ist, so verweise ich auf die beigefügte Zeichnung und constatire die a nastomotische Verbindung der Pleurazweige der Pulmonalis und Bronchialarterien.

Um nicht missverstanden zu werden, muss ich hier noch anführen, dass bei jeder gelungenen Injection der Lunge, von der Aorta aus, Bilder zu Stande kommen, die diese anastomotische Verbindung beweisen - ich habe dieses etwas umständliche Vorgehen hier angefïhrt, um den Beweis zu liefern, dass sowohl die Bronchial-, als auch die Pulmonalarterie miltelst der zur Pleura mediastinalis 
tretenden feinen Arterienzweige gefüllt werden können. Embryonale menschliche Lungen und die Neugeborner sind ein vortreffliches Injectionsmaterial für die in Rede stehenden Untersuchungen ibres bedeutenden subpleuralen und interalveolären Bindegewebslagers wegen.

Was die Anastomosen der Arteria bronchialis anbetrifft, so hat Hyrtl ${ }^{1}$ ) nachgewiesen, dass sie entweder im ersten Theilungswinkel des Bronchus, wenn ihrer mebr als eine vorhanden ist oder bereits extra pulmonem vorhanden sind, - dass die 2-3 Aeste derselben, die jeden grösseren Zweig des Bronchus begleiten, unter einander communiciren. Jede gelungene Bronchialarterieninjection giebt beweisende Bilder, als dass ich mich hier ein Weiteres auszulassen brauchte, - Hyrtl hat die Anastomosen mit Corrosionsmassen an menschlichen Lungen dargestellt (Corros.-Anatom. Tab. II, Fig. 1, c).

Das über die Aortenzweige der Arteria bronchialis und deren Verästelungen in der Lunge Mitgetheilte lässt sich in folgender Weise zusammenfassen:

1. Die Hauptstämme der Arteriae bronchỉales verästeln sich am Bronchus, - am Alveolargange haben die Stämmchen als solche aufgehört, ihre Capillaren hängen mit denen der Pulmonalarterien zusammen. Der grössere Theil der spärlichen Seitenäste versorgt das submucöse, peribronchiale und perivasculäre Bindegewebe, die Nerven, Lymphgefässe etc., der kleinere Theil tritt zu den Alveolen anderer Bronchialsysteme und löst sich daselbst capillär auf.

2. Die mit der Pleura mediastinalis zur Lunge tretenden Stämmchen breiten sich in der Pleura, im interlobulären Bindegewebe aus, versorgen die subpleural und interlobulär verlaufenden mächtigen Lymphgefässe, lösen sich aber auch an den Alveolen und Infundibeln capillar auf.

3. Es anastomosiren die Pleura- und Bronchialarterien, theils untereinander, theils mit Zweigen der Arteria pulmonalis.

b. Bronchialgefásse, die aus der Pulmonalarterie stammen.

Wenn man die eine Lunge eines eben durch Verbluten getödteten Thieres (Hund, Kaninchen) vor der Aorta, die andere von der Arteria pulmonalis aus injicirt, so dringt bei der ersteren verbältnissmässig wenig Injectionsmasse in die Gefässe der Mucosa des Bronchus vor, während im Lungenparenchym wenigstens fleckweise

1) Hyrtl, Corrosionsanatomie. S. 95 u. 96. 
reichliche Injectionen nachweisbar sind. Dagegen zeigt die Mucosa der anderen Lunge nicht nur eine intensive Färbung, sondern es ist die Injectionsmasse in das Lumen der Bronchien und Trachea ausgetreten. Bei Untersuchung dieser letzteren findet sich eine Füllung, sowohl der Bronchialarterien, als auch der sämmtlichen Gefässe der Mucosa neben einer vollständigen Füllung des Lungenparenchyms, also Infundibeln und Alveolen. Dieses leichte und massenhafte Eindringen der Injectionsmasse in das angebliche Gebiet der Bronchialarterie ist eine gewiss bemerkenswerthe Thatsache. Angaben über dieses Verhalten finden sich bereits in der Literatur. Fr. Arnold (l. c.) hebt z. B. hervor, dass die feineren Zweige der Lungenschlagader zu den Bronchien treten, - Hyrtl (1. c.) hat bei Injectionen der Pulmonalarterien eine Füllung der Gefässe der Bronchialschleimhaut gefunden, - H en le sagt „die Pulmonalarterie breitet sich in der Bronchialschleimhaut aus" (1. c.), - Luschka (l. c.) giebt bestimmt an, dass Zweige der Pulmonalarterie zur Bronchialschleimhaut sich begeben und sich in dieser zu einem Capillarnetz auflösen.

Bei einer einigermaassen gelungenen Injection der Bronchialgefässe von der Arteria pulmonalis' aus findet man einen ausserordentlichen Reichthum. injicirter Gefässe; es liegen am Bronchus, im Gegensatze zu dem Principe der Gefässanordnung im Lungengewebe, wie ich mich überzeugte, Zweige, die aus der Arteria pulmonalis stammen, neben solchen, die zur Vena pulmonalis gehen, - Zweige der Arteria bronchialis neben Zweigen der Vena bronchialis. Und doch kommt es darauf an, nachzuweisen, welche Zweige namentlich von der Arteria pulmonalis herrühren, welche namentlich zur Vena pulmonalis gehen, wie der Verlauf der einen, wie der der anderen, welches das Gefässgebiet der Arteria pulmonalis, welches Gefässgebiet die Arteria bronchialis inne hat. Vor allen Dingen aber kommt es darauf an, den Zusammenhang 2 wischen den grossen Stämmen der Arteria pulmonalis a $\mathrm{m}$ Bronchus mit Zweigen derselben in der Bronchialwand klar vor sich zu haben.

Der vielen capillaren und vorcapillaren Anastomosen wegen nützen weder einfache noch Doppelinjectionen, mit Silberlösungen erreicht man des starken Epithellagers wegen nichts. Ich empfehle deshalb die Hoyer'sche Corrosionsmasse, - je nachdem man sie mehr oder weniger eindickt, warm oder kalt injicirt, mit absolutem 
oder schwach wasserhaltigen Alkohol den Schellack löst and einspritzt, gelingl es, entweder nur Capillaren oder auch die kleinsten Gefässe auszuschalten. Da ich die Masse nicht für Corrosionspräparate, sondern für Schnittpräparate verwandte, so füllte ich vor der Injection der Gefässe den Bronchialbaum mit warmem Leim; ich dehnte die Bronchien auf diese Weise entsprechend aus und erhielt objecte, die sich besser schneiden liessen. Ich empfehle auch hier embryonale menschliche Lungen, ebenso Lungen Neugeborner; bei Untersuchung der zur Bronchialwand tretenden Aeste der Pulmonalarterie injicirte ich diese vom Stamm aus, - bei Untersuchung der zur Vena pulmonalis tretenden Venen des Bronchus injicirte ich erstere vom linken Atrium aus.

Bei diesem Vorgehen konnte ich mich nun davon überzeugen, dass die Pulmonalarterien starke Aeste zur Wand des Bronehus entsenden. Diese durchdringen die Faserhaut, die Submucosa und treten ziemlich gesammelt zur Mucosa, - verlaufen hier in den longitudinalen und parallel zu einander gestellten Falten der grösseren Bronchien, anastomosiren vielfach untereinander und senden pinselförmig sich auflösende Zweige, welche bis an die Membrana basilaris herantreten und sich dicht an dieselbe anlehnen. In den faltenlosen Bronchien trifft man ein Gefässnetz, dessen Zweige in mehreren Schichten übereinander gelegen sind und durch rechtwinklig abgehende Seitenzweige in Verbindung stehen. Mit dieser Injectionsmethode liess sich somit constatiren, dass ein vorwiegend grosser Theil der Pulmonalarterienzweige zur Mucosa und zur subepithelialen Schicht dringt, auf diesem Wege aber wenig Zweige abgiebt. Zur Untersuchung der weiteren Verästelung benutze ich Injectionen mit farbigen wässerigen oder leimigen Lösungen. In der subepithelialen Schicht findet sich ein Capillarnetz mit engen Maschen und weiten Röhren, das in seiner Configuration Aehnlichkeit mit denjenigen der Alveolenwände hat. Eigenthümlich sind an diesen subepithelialen Capillarnetzen die ampullenartigen Auftreibungen derselben, welche den Gefässen eine gewisse Aehnlichkeit mit Lymphgefässen verleihen. Die Durchmesser der Capillaren dieser subepithelialen Lage betragen $0,005 \mathrm{Mm}$., diejenigen der ampullären Anschwellungen $0,026 \mathrm{Mm}$.

Ich habe wiederholt erwähnt, dass bei der Injection der Pulmonalarterie die Injectionsmasse in das Lumen der Bronchien ein- 
dringe. Bei der milkroskopischen Untersuchung solcher Objecte findet man dieselbe zwischen den Epithelien des Bronchus; man kann aber überdies die Bahn verfolgen auf weleher die Injectionsmasse vom Gefäss aus in die Epithelschicht vorgedrungen ist: man trifft nehmlich an den Gefässen und ausschliesslich an den dem Bronchiallumen zugewendeten Flächen kleine, durch ausgetretene Injectionsmasse erzeugte dreieckige und lineäre Figuren, welche mit den zwischen den Epithelien gelegenen Massen continuirlich zusammenhängen; es erinnern diese feinen Zeichnungen auffallend an Kittleistenzeichnungen, die bei Aspiration von Indigcarmin zwisehen den Epithelien zu Stande kommen.

Mit wenigen Worten möchte ich hier noch der Gefässverzweigungen in der Trachealschleimbaut gedenken. Die Arteriae tracheales lösen sich zwischen je $z$ wei Knorpelringen in mächtige, die Breite des Lig. interanulare baltende Gefässbuchten auf und anastomosiren durch kurze, dicht gelagerte und ampullär erweiterte Gefässe. Ueber der Membrana transversa, dem ligamentösen Theil der Trachea, verjüngen sie sich und bilden ein dichtes, in mehreren Schichten übereinander liegendes Netz.

Bezüglich der zum Bronchus tretenden Zweige der Arteria pulmonalis wäre somit hervorzuheben, dass sie, ohne an die äusseren Schichten des Bronchus besondere Aestehen abzugeben, bis zur Membrana basilaris rordringen, in der subepithelialen Schicht gemeinsam mit den verhältnissmässig spärlichen Zweigen der Arteriabron. chialis ein dichtes Capillarnetz bilden.

c. Zweige der Bronchialgefässe, die zu deo Pulmonalvenengehen.

Bei der Füllung der Lungengefässe, vom linken Atrium aus, dringt die Injectionsmasse nicht nur in die Lungenvenen und von da in die an den Infundibula sich verästelnden Zweige der Arteria pulmonalis, sondern es füllen sich auch in der Broncbialmucosa befindliche Gefässe. Sie umspinnen die einspringenden Schleimhautfalten und entstehen aus dem subepithelialen Gefässnetze. Es sind nicht kleine Aeste, die mit den Pulmonalvenen zusammenhängen resp. sich in dieselben einsenken, sondern bedeutende Gefässzweige, deren Durchmesser beträchtlicher ist als derjenige der zum Bronchus tretenden Zweige der Pulmonalarterie (Fig, 9). (Ichs 
habe auch hier für das Erkennen der gröberen Zweige mich der Hoyer'schen Masse bedient, - zur Injection der capillaren Netze, die sich an die Basilarmembran anlehnen und auch interepitheliale Extravasate bilden, brauchte ich wässerige oder leimhaltige farbige Massen.)

Aehnliche Beobachtungen werden von Hyrtl (l. e.) und Luschka mitgetheilt; der erstere sagt, dass die „Bronchialgefässe zum Theil ihr Blut in die Babn der Vena pulmonalis ergiessen"; Luschka (l. c.) giebt an, "dass fast alle Venchen als sehr kleine Aeste in die Pulmonalvenen entlang dem Verlaufe derselben sich einsetzen". Es läge sehr nahe die Beobachtung, dass aus dem Bronchus abtretende Gefässe in die Pulmonalvenen sich einsenken, in dem Sinne der respiratorischen Function der Bronchialschleimbaut zu verwerthen; ich ziehe es zunächst vor, mich mit der Feststellung der interessanten Thatsache zu begnügen.

d. Zweige der Bronchien und der Lungen, die zur Azygos und Cava superior gehen.

Injicirt man einem eben getödteten Hunde in die Aorta gefärbten Leim, nachdem man den Bronchialbaum mit concentrirter Silberlösung gefüllt hat, so kann man nachweisen, dass die Injectionsmasse durch das System der Bronchial- und Pleuraarterien bis zum rechten Atrium vordringt. Die mikroskopische Untersuchung ergiebt, dass ausser den im.interlobulären und subpleuralen Gewebe gelegenen Zweigen der Pleura und Bronchialarterien ein System von Gefässen sich gefüllt hat, die den gleichen Verlauf einhalten wie die ersteren, nur weiter sind und unverkennbar den Charakter von Venen besitzen. Wird die Injection von der Vena azygos aus unternommen, so erreicht man gleichfalls eine Füllung dieser beiden Gefässsysteme und trifft die Injectionsmasse in der Aorta. Es kann demnach kein Zweifel bestehen, dass dem System der Pleura und Bronchialarterien ein ähnliches der PIeura und Bronchialvenen entspricht, welches sein Blut in die Azygos und Cava superior ergiesst.

Auf Grund der mitgetheilten Beobachtungen lassen sich über die Verzweigungsgebiete der einzelnen Gefässsysteme folgende übersichtliche Zusammenstellungen geben:

1. Die Aeste der Arteria pulmonalis verbreiten sich wesentlich und in ausgedehntester Weise an den Infundibeln und Alveolen; 
ausserdem aber in der Mucosa der Bronchien. In das interlobuläre und subpleurale Bindegewebe treten mehr vereinzelte Zweige ein. Die Arteria pulmonalis stebt somit zwar vorwiegend, aber nicht ausschliesslich einer secretorischen Function vor.

2. Die sogenannten Arteriae bronchiales verzweigen sich vorwiegend in den äusseren Lagen der Bronchialwand, dem subpleuralen und interlobulären Bindegewebe; sie entsenden aber auch Aestchen zu den Alveolen und zur Mucosa der Bronchien. Die Arteriae bronchiales sind somit vorwiegend aber nicht aus schliesslich nutritive Gefässe.

3. Die aus dem Capillarbezirke der Infundibeln und der Mucosa der Bronchien entspringende Gefässe ergiessen inr Blut hauptsächlich in die Pulmonalvenen, während diejenigen Gefässe, welche aus den in den äusseren Schichten der Bronchialwand, sowie im subpleuralen und interlobulären Bindegewebe gelegenen Capillarbezirken stammen, als sogenannte Bronchialvenen in die Azygos und Cava superior eintreten.

4. Berücksichtigt man die Verbreitung der Gefässe in den einzelnen Gewebstheilen nach ibrem genetischen Ursprung aus den Keimblättern, so würde sich als Regel ergeben, dass die Abkömmlinge des Drüsenblattes, mögen sie sich nun zu einem Plattenepithel in den Alveolen umgestalten oder als Gylinderepithel in den grossen Bronchien erhalten, wesentlich von den $Z$ weigen der Arteria pulmonalis, diejenigen des Darmfaserblattes von den Arteriae brouchiales versorgt werden.

Aus dieser Zusammenstellung ergiebt sich aber ferner eine bemerkenswerthe Thatsache, welche mir von grosser Bedeutung zu sein scheint, dass die Gebiete der einzelnen Gefässe keineswegs so begrenzte sind, wie dies neuerdings von Cohnheim und Litten angenommen worden ist. Was speciell die Arteria pulmonalis anbetrifft - so stehen deren Zweige unter sich in ausgedehnter Verbindung; wenn diese auch wesentlich capillärer Natur ist, so haben wir gesehen, dass dergleichen capilläre Verbindungen unter gegebenen Verhältnissen zu weiten anastomotischen Zweigen sich umgestalten können. Ausserdem aber bestehen zahlreiche Verbindungen, arterielle Anastomosen zwischen der Arteria pulmonalis und den Arteriae bronchiales; was ihre capillären Verbindungen an den Uebergängen der Bronchien in die Alveolengänge anbetrifft, so 
würden diese von $14 m$ so grösserer Bedeutung sein, als die Aeste der Bronchiales unter sich durch arterielle Anastomosen verbunden sind. Wenn somit feststeht, dass man die Arteria pulmonalis in so fern als „Endarterie“ auffassen kann als ihre Zweige unter sich nur durch capilläre Röhren communiciren - so ist doch auf der anderen Seite kaum zu verkennen, dass in Anbetracht der Anwesenheit zahlreicher capillärer Anastomosen zwischen ihren Zweigen und denen der Arteriae bronchiales ein sehr leichtes Ueberströmen des Blutes aus dem einen Gefässsystem in das andere ermöglicht wird, zumal wenn arterielle Anastomosen zwischen beiden ausserdem noch vorhanden sind. Die Thatsache aber, dass die Capillaren jeder Zeit zu weiten Gefässzweigen sich umgestalten können, muss uns daran zweifeln lassen, dass der Arteria pulmonalis in functionellem Sinne die Bedeutung einer "Endarterie" zukomme.

Es soll die Aufgabe des experimentellen Theiles sein, weiteres Material für die Beurtheilung dieser wichtigen Fragen zu liefern.

\section{Experimenteller Theil.}

In dem ersten Theile ist auf dem Wege der anatomischen Untersuchung der Nachweis geführt worden, dass die Bezirke der in den Lungen sich vertheilenden Gefässe keineswegs so abgeschlossen sind, wie dies in der neueren Zeit von verschiedenen Seiten geltend gemacht worden ist, dass vielmehr die einzelnen Zweige der Arteria pulmonalis und Arteria bronchialis unter sich in vielfacher wechselseitiger Beziehung stehen. So bedeutungsvoll diese Erfahrung an und für sich ist, so darf andererseits doch nicht verkannt werden, dass man nicht berechtigt ist, aus der anatomischen Anơrdnung der Gefässbahnen direct auf die Blutvertheilung unter pathologischen Verhältnissen zu schliessen. Cohnheim und Litten waren vollkommen im Rechte, wenn sie für die Beurtheilung dieser Verhältnisse dem Experimente eine grössere Bedeutung vindicirten.

Wie schon früher erwähnt, war Virchow der Erste ${ }^{1}$ ), der diesen Weg betreten hat. Die von ihm angestellten Versuche haben zu dem Ergebniss geführt, dass in der That die Bronchialarterie

1) Virchow, Gesammelte Abhandlungen. S. 295. 
für das nutritive Geschäft der Lunge genïge und dass das functionelle Gefäss, die Arteria pulmonalis, ohne Nachtheil für die Ernährung des Organs unterbrochen werden könne. $\mathrm{Er}_{\mathbf{r}}$ fand, dass nach Verstopfung der Lungenarterie ein collateraler Kreislauf durch die Bronchial- und Intercostalarterie hergestellt werde, und "dieser Kreislauf sich nicht aus dem Gebiete der Lungenarterie, sondern aus der Aorta durch Broncbial- und Intercostalarterien entwickele " ${ }^{1}$ ) "durch Erweiterung präexistirender Gefässe" ${ }^{2}$ ). Virchow ging bei seinen Versuchen in der Weise vor, dass er in die Vena jugularis Pfröpfe einbrachte und das Thier nach Monaten tödtete. Er fand in einem dieser Fälle (Experiment XX, gesammelte Abhandlungen S. 295) eine vollständige Verstopfung der Pulmonalarterienzweige eines Lappens und eine entsprecbende Erweiterung der Bronchialund Intercostalarterien.

Gohnheim und Litten ${ }^{3}$ ) halten dies Experiment nicht für beweiskräftig, weil in der Zeit seit Einbringung der Pfröpfe neue Gefässe entstanden sein können. Dieselben betonen, dass für die Beurtheilung des Zustandes der Gefässbahnen un mittelbar nach der Embolisirung, wo doch solche Gefässramificationen noch nicht vorhanden waren, der Versuch keinen Aufschluss gebe. Um diesen Zweck zu erreichen, haben die genannten Autoren einen anderen Weg eingeschlagen. Nach theilweiser Resection der 2, und 3. linken Rippe wurde der zur linken Lange tretende Stamm der Arteria pulmonalis unterbunden und dann in die Arteria femoralis des lebenden Kaninchens in Wasser lösliches giftfreies Anilin peripher eingespritzt. Cohnheim und Litten baben nun gefunden, dass in solchen Fällen die intacte Lunge eine intensiv blaue Färbung annimmt und bei der mikroskopischen Untersuchung die sehönste Füllung aller Gefässe zeigt, während die Lunge, deren Pulmonalis unterbunden war, völlig blass bleibt und nur die Bronchialschleimhaut sich intensiv blau färbt, gleich derjenigeu der anderen Seite. Aus diesem Versuchsergebniss wird von C. und L. der Schluss gezogén, "dass die Bronchialarterien dem Lungenparenchym selbst. nicht geringe Quantitaten Blutes zuführen und dass sich dies auch nicht ändert, wenn die Pulmonalwege verschlossen sind“. Bei

1) Dieses Archiv Bd. I. S. 18.

2) Dieses Archiv Bd. III. S. 456.

3) Cohnheim und Litten, Dieses Archiv Bd. 65. S. 103. 
einer zweiten Reihe von Versuchen wurden Kaninchen und Hunden in gewöhnlicher Weise Paraffinemboli in die Vena jugularis eingebracht und später vorsichtig Aufschwemmungen von cbromsaurem Bleioxyd nachgespritzt. Das Ergebniss dieser Versuche war, dass die Bleisalzpartikél niemals in demjenigen Lungenabschnitt getroffen wurden, welcher jenseits der obturirten Arterienstelle gelegen war. Das Resultat wird von C. und L. als durchaus eindeutig bezeichnet: „Die arteriellen Verästelungen der Pulmonalis anastomosiren nirgend mit einander, es sind Endarterien". Die Annahme einer ausschliesslich nutritiven Function der Arteria bronchialis, einer ausschliesslich functionellen Bedeutung für die Arteria pulmonalis sind die weiteren Consequenzen.

Durch die Schlüsse, welche C. und L. aus ihren Versuchen ableiten, wird die Möglichkeit der Herstellung eines collateralen Kreislaufs in der von Virchow angenommenen Weise in Frage gestelit: ein Resultat, das für mich um so überraschender sein musste, als meine Untersuchungen am todten und lebenden object mich hatten Anordnungen der Gefässbahnen kennen lernen, von denen man hätte voraussetzen sollen, dass sie der Einleitung eines collateralen Kreislaufs sofort nach Embolisirung eines Gefässes günstig seien. Da ich mich aber der Richtigkeit des Satzes, dass man aus der anatomischen Anordnung der Gefässbahnen noch nicht auf Verhältnisse bei Circulationsstörungen zu schliessen berechtigt ist, nicht zu entzichen vermochte, so unternahm ich eine Wiederholung der von C. und L. gemachten Versuche. Im weiteren Verlauf meiner Arbeiten sah ich mich genöthigt, die Versuchsanordnungen in der verschiedensten Weise zu modificiren. Die Versuchsreihen, die ich anstellte, sind folgende:

1) Einfache Unterbindungen verschiedener Lungengefässe, ohne gleichzeitige Injection oder Infusion gefärbter Flüssigkeiten.

2) Unterbindungen der Lungengefässe bei gleichzeitiger Einführung von Anilinlösungen in das Blut des lebenden Thieres.

3) Unterbindungen von Lungengefässen und Infusion von Zinnoberaufschwemmungen in die Vena jugularis.

1. Einfache Unterbindungen der Lungengefässe.

Zunächst nahm ich Unterbindungen der Arteria pulmonalis vor, dann ging ich zu Unterbindungen der Vena pulmonalis über. 
Bei einer weiteren Versuchsreihe wurden Pulmonalarterie und Venen gleichzeitig und endlich bei einer letaten Serie von Versuchen Arteria pulmonalis, Venae pulmonales, die Zweige der Arteria bronchialis, welche von der Art. intercostalis prima entspringen, unterbunden. Der Zweck dieser Versuche war der, die Veränderungen in der Blutvertheilung kennen zu lernen, welche nach diesen verschiedenen Unterbindungen eintreten. Es handelte sich dabei wesentlich nur um Constatirung gröberer Verhältnisse. Ich wählte fettarme, junge Hunde und Kaninchen; die Unterbindungen sind. bei den letzteren leichter auszuführen als bei ersteren, - Hunde gingen bei der unbedeutendsten Eröffnung "der Pleura, ohne dass ich die Ursache des plötzlichen Todes mir zu erklären im Stande war, ungemein häufig zu Grunde.

a. Unterbindung des gemeinschaftlichen Stammes der Art. pulm. der linken Lunge.

Die Arterie wurde von dem darunterliegenden Bronchus und den beiden über sie hinziehenden Venae pulmonales der oberen Lappen isolirt und unterbunden. Einige Versuchsthiere wurden eine oder wenige Stunden darauf getödtet, andere liess ich 12,24 , 48-120 Stunden leben. Je nach der Zeit, welche zwischen der Operation und dem Tode verstrichen war, fanden sich an den Lungen verschiedene Veränderungen. Lungen, die eine Stunde nach der Operation untersucht wurden, waren collabirt, blutroth und etwas welk. Schon nach 5 Stunden zeigten sich intensivere Veränderungen an ihnen, - sie waren nicht nur dunkelroth gefärbt, sondern auch an manchen Stellen hämorrhagisch infarcirt, die Alveolen und Alveolargänge mit Blutkörperchen ïberfüllt. Nach zwei Tagen begannen sich meist Veränderungen der Pleura und des Pericards einzustellen, die im Verlauf von $4-5$ Tagen den Charakter eitriger Entzündung annahmen, - wahrscheinlich Folgen des operativen Eingriffs. Bemerken will ich noch, dass in $15 \mathrm{Ver}$ suchen mit vollständiger Unterbindung der Arteria pulm. nicht ein einziges Mal eine brandige Veränderung der Lunge sich einstellte. Um so häufiger, nehmlich in 7 Fällen, fand ich pneumonische Affectionen verschiedenen Grades. Eine hochwichtige Thatsache aber bestand in allen diesen Fälen darin, dass der intrapulmonale Stamm der Art. pulm. sowohl als aueh die 
grösseren $Z$ weige desselben mit einem Blut- und Faserstoffgerinnsel erfült, der je nach der Dauer des Versuches mehr oder weniger derb war. Auch in den Stämmen der Venae pulmonales war Blut enthalten: dasselbe war aber entweder flüssig oder frisch geronnen, wie in den Zweigen der Art. pulmon. 3.-4. Ordnung. Bezüglich des Verhaltens der Bronchialgefässe will ich hervorheben, dass die in dem subpleuralen und interlobulären Gewebe, sowie die in den äusseren Schichten der Bronchialwand verlaufenden Gefïsse stärkere Füllung zeigten, während die Mucosa der Bronchien meist blass war.

Bei der Untersuchung der Lungen wurde in allen Fällen mit einer Injection des vor der Unterbindungsstelle gelegenen Abschnittes der Pulmonalarterie vom Herzen aus eingeleitet, nur um zu constatiren, dass die Unterbindung der Art. pulmonalis eine vollständige war.

Das Ergebniss der eben angeführten Versucbsreihe wäre somit folgendes: in Lungen, bei denen der Hauptstamm der Art. pulm. unterbunden wurde, kamen nach kurzer Zeit starke Blutüberïullungen und selbst hämorrhagische Infarcirungen zu Stande.

b. Unterbiadungen des Stammes der Arteria pulmonalis nebst den über diesen wegziehenden beiden Venen.

$\mathrm{Zu}$ einer gleichzeitigen Unterbindung dieser Gefässe wurde ich dadurch veranlasst, dass bei den unter a. geschilderten Versuchen die Venen mit Blut überfüllt waren; die Möglichkeit, dass es sich hier um ein Zuströmen des Blutes durch diese zu den Lungen handeln könne, liess sich um so weniger von der Hand weisen, als auch Cohnheim und Litten in den grössten Venen, blaue Schollen" getroffen batten, von denen sie annehmen, dass sie aus dern Vorhof übergetreten seien. Da es sich bei diesen Versuchen weniger um die Kenntniss der Folgezustände als vielmehr um diejenige der Blutvertheilung, der Zufuhr handelte, wurden die Thiere stets wenige Stunden nach vorgenommener Unterbindung getödtet. Bei der Section erschienen die oberen Lappen, d. h. die Lappen deren Venen unterbunden waren dunkelroth und hämorrhagisch infiltrirt, der untere Lappen meist blass. Bej der Entfernung der Ligatur an den oberen Venen entleerte sich aus diesen reichliches 
flüssiges Blut. Auch in diesen Fällen enthielten der Stamm und die grösseren Zweige der Art. pulm. Blut und Fibringerinnsel, die Gefässe 3.-4. Ordnung waren mit Blut gefüllt, ebenso die Alveolen und Alveolargänge.

Aus a. und b. liess sich folgern, dass trotz abgebundener Art. und Ven. pulmonalis der Lunge reichlich Blut zugeführt wurde.

c. Unterbindung des Stammes der Art. pulmonalis und der oberen Lungenvenen mit einer Ligatur, gleichzeitige Abklemmung der unteren Vene.

Zur einfachen Abklemmung der letzteren war ich genöthigt, weil dieselbe mit einer rechten unteren Pulmonalvene in einer gemeinschaftlichen Falte der Pleura mediastinalis liegt und bei einer Verletzung dieser es zu Eröffnung des rechten Pleuraraumes und augenblicklicher Erstickung gekommen wäre. Bei Untersuchung der Lunge, nach Ablauf einer Stunde etwa, fand ich diese voluminös, prall und dunkelroth gefärbt. Aus der abgeklemmten und den unterbundenen Venen entleerte sich viel Blut worauf die Lunge ihre pralle Beschaffenheit verlor. Der Stamm und die grossen Seitenzweige der Art. pulm. enthielten Blut und Faserstoffgerinnsel. Wie bei den Arterien so war auch bei den Venen das Blut vorwiegend in den grösseren Zweigen; die kleineren Gefässe waren relativ leer und schienen sich einen freien Abfluss bewahrt, - der Lungenkreislauf sich auf Arterien und Venen kleineren Calibers beschrärukt zu haben. Die Bronchialmucosa erschien nicht hyperämisch, dagegen waren die grösseren in den äusseren Schichten der Bronchialwand gelegenen Gefässstämme blutreich und dilatirt, ebenso die im Ligamentum suspensorium des unteren Lappens vorhandenen Gefässe; ja es war zwischen beiden Pleurablättern selbst zu Hämorrhagien gekommen. Um mir eine Uebersicht der zuletzt erwähnten Gefässe zu verschaffen, dehnte ich die dünne Membran in der Weise aus, dass ich den Bronchus und den Oesophagus mit Leim fülte. Man konnte dann in der gespannten Membran sehr leicht die dilatirten Gefässe nachweisen.

Die bisher geschilderten Versuchsreihen haben zu dem Ergebniss gefürt, dass auch bei unterbundenem Stamme der Arteria pulmonalis die Lunge im Zustande beträchtlicher Blutfülle getroffen wird, - diese kommt auch in Lungen zu 
Stande, deren Pulmonalvenen unterbunden sind, in dem letzteren Falle ist die Blutüberfüllung eine stärkere. Trotz der unterbundenen Venae pulmonales kommt aber der Abfluss zu Stande. Dass bei der letzten Versuchsanordnung die Blutzufuhr nur durch die Arteriae bronchiales bewerkstelligt wurde, ist ein gewiss gerechtfertigter Schiuss, der überdies noch durch den Nachweis einer Dilation derselben thatsächlich gestützt wird.

\section{d. Unterbindung des Stammes der Arteria pulmonalis der beiden oberen Pulmonalvenen und des Bronchus mit einer Ligatur.}

Nachdem es mir gelungen war, den Nachweis zu führen, dass bei unterbundener Pulmonalarterie durch die Bronchialarterie der Lunge Blut zugeführt werde, so handelte es sich noch darum, festzustellen, ob die einzelnen aus verschiedenen Stämmen entspringenden Arteriae bronchiales (Pleurae) im Stande wären für einander vicarïrend einzutreten. Da die aus der Intercostalis prima kommenden Bronchialarterien zum untersten Ende der Trachea treten und sich mit den beiden Bronchien in die Lungen einsenken; so war ein Verschluss derselben am leichtesten in der Weise zu erreichen, dass man den Broncbus, nach Eröffnung und Schonung der ihn umhüllenden Pleura mediastinalis, abband neben Unterbindung der Art. pulmonalis und Venen. Die Zufuhr war nunmehr nur noch mittelst der Arteriae tracheooesophageae, pericardiophrenicae, pleurae mediastinalis etc. etc. möglich. Als ich nach zwei Tagen die Untersuchung vornahm, fand ich den oberen Lappen roth, gespannt und hämorrhagisch infiltrirt, den unteren, bei dem die Vene nicht abgebunden war, blasser; die subpleuralen und mediastinalen Gefässe waren mit Blut gefüllt, der Stamm der Art. pulm. und seine nächsten Zweige hatten den steten Fibrinpfropf. Aus diesen Versuchen scheint mir hervorzugehen, dass bei Verschluss der aus der Intercostalis entspringenden zum Bronchus tretenden Arterienzweigen neben Unterbindung der Arteria pulmonalis eine Blutzufuhr mittelst der feinen Arterienreiser der serösen Häute (Pleura-PericardiumMediastinum) zu Stande komme.

Um meine Versuchsreihe annähernd vollständig zu machen unterband ich in einem Falle nur die Venae pulmonales. Der Abfluss war durch die Azygos und Vena cava superior ermöglicht.

Vergleiche ich diese Versuchsreiben mit anatomischen That- 
sachen, die ich durch Injectionen todter Objecte erhalten hatte, so finde ich eine Uebereinstimming beider: ein Collateralkreislauf kommt, sofort nach Unterbindung der Arteria pulmonalis, durch Erweiterung präexistirender Gefässe zu Stande; die Bronchialarterie tritt für die verlegte Pulmonalarterie aushelfend ein.

2. Unterbindung der Arteria pulmonalis und nachfolgende Einführung von Anilinlösung in das Blut des lebenden Thieres.

Zunächst hielt ich mich genau an die von Cohnheim und Litten gegebenen Vorschriften, indem ich Lösungen von giftreiem, in Wasser löslichem Anilinblau peripher in die Arteria femoralis einspritzte. Die Anilinlösungen enthielten immer 0,5 pCt. Kochsalz. Ich bezog das Anilinblau von Meister, Lucius und Bruning in Höchst, später von Tromsdorff (B. 0. 2. 3. 4. 5).

Den ersten Versuch führte ich in der Weise aus, dass ich einem jungen Hunde, nachdem ich die Arteria pulmonalis s. unterbunden hatte, bis zu $500 \mathrm{Ccm}$. Anilinlösung peripher in die Art. femoralis innerhalb einer Zeit von etwa zwei Stunden injicirte. Trotz der Menge von Injectionsmasse war das Thier kaum blau gefärbt, der Schenkel ödematös und die Musculatur intensiv blau; nur mit ausserordentlicher Mühe gelang es einzuspritzen: beide Lungen waren schwach blau.

Da das Resultat dieser Versuche so wenig befriedigend ausfiel, änderte ich dieselben in der Weise $a b$, dass ich Kaninchen bis zu $150 \mathrm{Ccm}$. Anilinlösung in centraler Richtung in die Arteria femoralis einspritzte und dieselben nach einer Stunde tödtete. Dabei fiel mir auf, dass die blaue Färbung erst mit dem Erkalten des Versuchsthieres eintrat. Die Lunge, an der die Pulmonalarterie durchgängig gelassen war, erschien fleckig aber saturirt blau; ihre Gefässe waren an diesen Stellen mit blauen Schollen gefüllt, namentlich die kleineren; die Bronchialmucosa erschien tiefblau. An der Lunge mit unterbundener Pulmonalarterie war oberflächlich keine blaue Färbung wahrzunehmen. Dagegen enthielt die Arteria pulmonalis von der Ligaturstelle an ein blaugefärbtes Gerinn$\mathrm{sel}$, das sich bis in die Verzweigungen 2.-3. Ordnung fortsetzte. Die kleineren Zweige und Capillaren enthielten äusserst sparsame, kleine blaue Flecken. Die Mucosa der Bronchien blieb blass. 
In einer weiteren Reihe von Versuchen infundirte ich Kaninchen nach Unterbindung der Art. pulm. 200-300 Ccm. giftreie Anilinkösung in die Vena jugularis externa. Die Infusion dauerte von 3-7 Stunden, d. h. ich hörte mit dem Tode des Thieres, der meist in einem soporösen Zustande eintrat, zu infundiren auf. Die Section folgte unmittelbar nach dem Tode des Thieres. Die Lungen mit unterbundener Art. pulm. waren auch in diesen Fällen äusserlich farblos; die Arteria und Venae pulmonales enthielten blau gefärbte Gerinnsel, die um so derber waren, je länger die Infusion gedauert batte; die Mucosa der Bronchien war blass. In der Lunge mit freier Art. pulm. war die Bronchialmucosa intensiv blau, das Lungenparenchym fleckig blau, die Oberfläche der Lunge blau und contrastirte ganz ausserordentlich mit der Lunge deren Arterie unterbunden war.

Um den Einfluss der Respiration auf die Blutvertheilung beurtheilen zu können, stellte ich Versuche an, bei denen dem Thiere möglichst grosse Mengen (bis $800 \mathrm{Cem}$.) Anilinlösung in die Vena jugularis infundirt wurde, während künstliche Athmung ausgeführt wurde. Ich muss hier constatiren, dass bei dieser künstlichen Athmung der Tod bedeutend später erfolgte - den Thieren somit grössere Quantität der Flüssigkeit infundirt werden konnte. Bei der Section solcher Thiere war die Haut hellblau gefärbt, die Bulbi prominirten stark, in der Bauchhöhle war ausserordentlich viel wässrige farblose Flüssigkeit enthalten, die Harnblase war wenig ausgedehnt, der Harn blass, dickflussig - die Harnentleerung während der Infusion äusserst spärlich; die Befunde an den Lungen waren im Allgemeinen dieselben, nur mit dem Unterschiede, dass die blauen Gerinnungen in den grösseren Verzweigungen und im Stamm der Art. pulm. bis zur Ligatur hin sich za einem festen, derben, intensiv blauen Blut- und Faserstoffperopf umgewandelt hatten.

Endlich wurden noch Infusionsversuche vorgenommen bei Kaninchen, denen ausser der Pulmonalarterie einzelne Venen, Bronchialarterien unterbunden waren. Ich hielt mich hierbei an dieselbe Aufeinanderfolge wie ich sie bei den einfachen Unterbindungen der Art. pulm. auseinandergesetzt habe. Die Ergebnisse stimmten im Wesentlichen mit denjenigen der anderen Versuchsreihen überein, zeigten aber die durch die Versucbsanordnung bedingten Verschiedenheiten. In den Fällen, in denen die Venae pulwonales mit 
anterbunden worden waren, enthielten diese nicht flüssiges, mit Anilinflocken durchmengtes Blut, sondern blau gefärbte, derbe Fibringerinnsel. Waren die Art. bronchiales mitsammt dem Bronchus unterbunden, so erschienen die collateralen $\mathrm{Zweige}$ dilatirt und gleichfalls mit blauen Schollen erfüllt.

Von den in den obigen Zeilen geschilderten Versuchsresultaten erfordern einzelne eine Erörterung. In den meisten Fällen war die Lunge, an der die Unterbindung der Art. pulm. ausgeführt wurde, entweder nicht oder nur sehr wenig, jedenfalls bedeutend geringer gefärbt, wie die andere Lunge mit durchgängiger Art. pulm. Es würde sich daraus ergeben, dass der ersteren weniger Farbstoff mit dem Blut zugeführt worden war: es ist selbstverständlicb, dass die Lunge mit unterbundener Art. pulm. nur wenig Farbstoff wird enthalten können, da die sämmtliche Infusionsflüssigkeit doch zunächst die Lunge mit durchgängiger Arterie passirt und viel Farbstoff zurücklässt und das um so mehr, wenn bewiesen werden konnte, dass bereits während des Lebens das Anilin sich in den Gefässen derselben in Schollen niederschlage oder absetze. Wenn aber Cohnheim und Litten angeben, dass in der Lunge mit unterbundener Art. pulm., mit Ausnahme der Bronchialmucosa und der Wurzeln der Pulmonalvenen, gar kein Farbstoff zu finden sei, so kann ich dem nicht beistimmen. Ich fand sehr häufig bei mikroskopischer Untersuchung, wenn auch vereinzelte und blasse, so doch unzweifelhafte Farbstoffpartikelchen in den Capillaren der. Alveolenwände, ganz abgesehen von dem in der unterbundenen Art. pulmon. befindlichen, zusammenhängenden, festen Blut- und Faserstoffgerinnsel, welches stets mehr oder weniger blau gefärbt war. Die Lungenvenen enthielten, wenn sie offen waren, mit blauen Schollen durchmengtes, flüssiges Blut; waren sie unterbunden, so fand sich ein mit Farbstoffpartikelchen durchsetztes, festes Gerinnsel. Es enthalten somit, nach meinen Beobachtungen, die Lungen mit unterbundener Art. pulm. gileichalls Farbstoffpartikelchen aber in geringerer Menge. Dieselben sind zuweilen im Lungengewebe so vereinzelt und so blass, dass sie sich nur bei mikroskopischer Untersuchung nachweisen lassen, zu der ich mich um so mehr aufgefordert fühlte als ich die blaugefärbten Fibringerinnsel in den Slämmen der Art. pulm. jedesmal vorfand. Bei Infusion geringerer Quantitäten mag 


\section{4}

es vorkommen, dass die Lungen wenig Farbstoff enthalten und dass sich derselbe der Beobachtung entziehe. Diese Farbstoffpartikel können aber nur durch die Bronchialarterien in die Lungen gelangt sein; die Annahme eines Eindringens von den Lungenvenen aus ist durch den Befund von Farbstoff in Lungen mit unterbundenen Venen ausgeschlossen. Dass die Zufuhr auf dem Wege der Bronchialarterien geschehe, ist um so wahrscheinlicher als dieselben sich bei der Untersuchung mit Farbstoff gefüllt zeigen und in Fällen, in denen die einzelnen Zweige unterbunden waren, die erweiterten collateralen Aeste solchen in grösserer Menge enthielten.

Noch in einem zweiten Punkte finde ich mich mit Cohnheim und Litten im Widerspruche; - dieselben erwähnen, dass die Mucosa der Bronchien, auch an Lungen, in denen die Art. pulm. unterbunden ist, eine blaue Färbung zeige, quantitativ ubbereinstimmend mit der der anderen Lunge. Bei einer grossen Anzahl von Infusionen (54), die ich ausführte, habe ich in keinem Falle an der Lunge mit unterbundener Pulmonalarterie eine gleich starke Färbung der Mucosa der Bronchien gesehen wie auf der anderen Seite, - sie contrastirte womöglich noch mehr mit der der anderen Seite, als es beide Lungen bereits thaten. Dieser Befund war mir verständlich, wenn $\mathrm{jch}$ die im anatomischen Theil gewonnene Erfahrung berücksichtigte, dass die Mucosa der Bronchien nicht nur von Zweigen der Arteria bronchialis, sondern hauptsächlich von Aesten der Art. pulmonalis, versorgt wird.

Bei den geschilderten Versuchen mit giftfreiem, in Wasser löslichem Anilinblau fiel mir auf, einmal die ungleiche Färbung in den einzelnen Versuchen, wenn auch nahezı gleiche Mengen infundirt worden waren, zweitens, dass die Färbung einige Zeit nach dem Tode an Intensität zunahm und endlich drittens, dass die besonders stark gefärbten Gefässbezirke immer eigenthümliche, colloide, schollenartige Partikel enthielten, die Füllung der Gefässe mit Farbstoff eine vielfach unterbrochene war. Diesem letzten Umstande musste ich es auch zuschreiben, dass meine Infusionspräparate fleckig, nur stellenweise mit Anilin gefüllt waren und sich, was Uebersichtlichkeil anbetrifft, kaum vergleichen liessen mit den künstlichen Injectionen todter Lungen. - Nicht unerwähnt darf ich ferner lassen, dass sich die Präparate in Alkobol nicht aufbewahren 
liessen, - sie wurden sehr bald blass - der Alkohol dagegen blau - mit anderen Worten das Anilinblau diffundirte. Es schien mir erforderlich, das Verhalten des Anilin im kreisenden Blute genauer za prüfen.

Wie bereits oben erwähnt, habe ich zu meinen Versuchen die verschiedensten Arten von giftfreiem, in Wasser löslichem Anilinblau verwendet; da meine Erfabrungen mit denen Cohnheim's und Litten's nicht stimmen wollten, sah ich mich gewissermaassen dazu genöthigt. Wesentlich verschieden waren bei diesen die Löslichkeitsverhältnisse. Auffallend war mir, dass selbst bei dem Präparat, welches sich als das am leichtesten lösliche erwies (1:600 bis $800 \frac{1}{2}$ procentiger Kochsalzlösung), selbst bei tagelangem Stehen Rückstände auf dem Filter blieben, die sich vollständig in Alkohol Jösten. So lösen sich z. B. von $1 \mathrm{Grm}$. Anilinblau (Tromsdorf B 2) in $600 \mathrm{Grm}$. $\frac{1}{2}$ procentiger Kochsalzlösung nur $0,6 \mathrm{Grm}$., 0,04 Grm. bleiben auf dem Filter und lösen sich in Alkohol. Ich konnte mich davon überzeugen, dass Alkohol ganz bedeutende Quantitäten Anilinblau zu lösen im Stande ist, dass die Löslichkeil desselben mit zunehmendem Gehalı der Flüssigkeit an Kochsalz abnimmt, dass das Anilinblau in etwa 5 procentiger Kochsalzlösung ungelöst bleibt. In diesen Lösungen bewahrte ich schliesslich meine mit Anilinblau injicirten Lungen.

Was das Verhalten des Farbstoffes im Blut anlangt, so geben C. und L. an, dass er in grösseren und kleineren Körnchen ausfalle, dass etliche Capillaren zeitweise oder definitiv verlegt wïrden, - selbst der Fall könne vorkommen, „dass das Anilin nicht über die Capillaren hina usgehe, nicht in das Aortensystem dringe". Auch ich habe ähnliche Schollenbildungen, Zusammenballungen gesehen und wahrgenommen, dass feinere Gefässzweige durch dieselben verlegt werden.

Um mir über diese Vorgänge Aufschluss zu verschaffen, nahm ich. Infusionsversuche am lebenden Frosch vor und beobachtete während derselben die Gefässe des Mesenteriums, dessen Oberfläche mit $1 \frac{1}{2}$ procentiger Kochsalzlösung berieselt wurde. Die Infusion führte jch in der Art aus, dass ich eine Lösung von Anilinblau (Tromsdorf B 2 1:600 einer $\frac{1}{2}$ procentigen Kochsalzsolution, filtrirt) tropfenweise in die Vena abdominalis ibberleitete und 2 war in der ersten Stunde $0,3 \mathrm{Ccm}$., in der zweiten $0,6 \mathrm{Gcm}$., in den folgenden 
bis $2 \mathrm{Ccm}$. Die Versuche wurden 7-8 Stunden lang fortgesetzt, so dass die Frösche $16-18 \mathrm{Ccm}$. im Ganzen infundirt erhielten. Schon nach Infusion von $0,15 \mathrm{Ccm}$. dieser äusserst lichtblauen Flüssigkeit traten, während das in den Gefässen kreisende Blut noch seine normale Farbe beibehielt, in den Capillaren blaue Wölkchen auf und legten sich gern dem. Theile der Gefässwand an, an welchen das Blut minder rapid vorbeiströmte. Sehr bald bildeten sich an den Gefässwänden spinnengewebige Niederschlăge, in denen sich rothe und weisse Blutkörperchen verfingen. Allmählich war die Wand des Capillarrohres in grösserer Ausdehnung mit lichtblauen Gerinnseln belegt, im eingeengten Flussbette oseillirte die Blutsäule, dann stockte sie; das ganze Gefässrohr war gefüllt und dann undurchgängig. Doch nicht nur an den Gefässwänden entwickeln sich primär diese Niederschläge, - auch an den Blutkörperchen treten sie als blaue Wölkchen auf, beschwerten dieselben, so dass sie sich träger bewegten. Im weiteren Verlauf der Versuche kam es bald $\mathrm{zu}$ intensiveren Kreislaufstörungen der Art, dass das Gefäss bald vorübergehend, bald dauernd durch Niederschläge verlegt wurde, die dann nicht mehr das Gefüge von Gerinnseln, sondern solider, lichtblauer, homogener Schollen angenommen hatten. In steter Aufeinanderfolge vollzogen sich solche Vorgänge schliesslich auch in grösseren Gefässen, die in ähnlicher Weise verstopft, ausgeschaltet wurden, - der Blutstrom sich collaterale Bahnen wäblte. Die Gerinnsel bildeten gewöhnlich nicht zusammenbängende, sondern vielfach unterbrochene Pfröpfe; zuweilen wurden diese wieder flott um sich an einer anderen Stelle des Gefässbezirkes einzukeilen. Bei längerer Dauer der Infusion traten in den lichtblauen Schollen dunklere Körnchen auf, ja selbst Niederschläge von deutlich krystallinischem Gefüge. Ich will schliesslich noch bemerken, dass auch an der Oberfläche des Mesenteriums fadenförmige, dasselbe weithin deckende, blau gefärbte Fibringerinnsel auftraten, dass selbst in den Fällen, in denen grössere Mengen der Anilinlösung infundirt worden waren, die Versuchsthiere oft erst 2 Stunden nach dem Tode eine intensiv blaue Farbe annahmen.

Die mit Anilinlösungen an Fröschen angestellten Versuche lebren, dass es nicht nur innerhab der capillaren Bahnen, sondern auch in grösseren Gefässen zur Bildung von Abscheídungen komme, welche diese theils vorübergehend, theils dauernd zu verlegen im 
Stande sind. Berücksichtigt man dieses Verhalten des genannten Farbstoffes im Blut, so wird man zugeben müssen, dass er zur Verwendung bei den in Rede stehenden Versuchen nicht geeignet ist. Einmal ist es bei der wechselnden Grösse der Pfröpfe zu erwarten, dass bald kleinere, bald grössere Gefässe durch diese Abscheidungen verlegt werden. Man wird somit nicht berechtigt sein aus der Vertheilung des Farbstoffes auf diejenige des Blutes oder gar auf die Configuration der Gefässbahn, Mangel oder Anwesenheit von collateralen Verbindungen zu schliessen. Insbesondere aber wird der Schluss unzulässig erscheinen müssen, collaterale Beziehung der Arteria pulmonalis und der Arteria bronchialis auszuschliessen, da es (insbesondere beim Kaninchen) in den engen Bronchialarterien sehr lejcht zu Verstopfungen kommen muss. Eine solche Vorsicht wird um so mehr geboten erscheinen, als, wie oben nachgewiesen wurde, auch in lungen, deren Arteria pulmonalis unterbunden war, Farbstoffpartikel gefunden wurden, nur in viel spärlicherer Menge als in derjenigen mit durchgängiger Arteria pulmonalis.

3. Unterbindungen der Lungengefässe mit nachfolgender Infusion von Zinnoberaufschlemmungen in die Vena jugularis.

Von der Richtigkeit der Cohnheim-Litten'schen Versuchsanordnung überzeugt, musste ich, nach den Erfahrungen, die ich mit den Anilinlösungen gemacht hatte, es mir angelegen sein lassen eine Infusionsfluissigkeit zu finden, von der vorauszusetzen war, dass sie "indifferent" sei, ,in den Gefässen bleibe ohne zu diffundiren", dass sie die gröberen Gefässe nicht verstopfe und selbst die feineren zu passiren im Stande sei. Bei der Anwendung dieser mussten sich richtige Aufschlüsse über die Blutvertheilung ergeben, Aufschlüsse die die anatomischen Befunde mit den experimentellen in Einklang zu bringen im Stande waren.

Nach vielen Versuchen ergab sich eine dünne Aufschwemmung von feinstem englischem Zinnober in $\frac{3}{4}$ procentiger Kochsalzlösung als das geeignetste Gemenge. Dieses wurde den Thjeren durch die Vena jugularis externa infundirt und zwar in erwärmtem Zustande von $36-39^{\circ} \mathrm{C}$. und continuirlich fliessendem Strome, - die Infusion dauerte $7-8$ Stunden, so dass die Quantität der Infusionsmasse eine 
sehr beträchtliche war (von $600-950 \mathrm{Ccm}$.). Bei einigen Versuchen wurde künstliche Respiration eingeleitet, bei anderen nicht; sie ist in den Fällen, in welchen eine Unterbindung aller Lungenvenen der linken Seite vorgenommen werden soll, nicht $\mathrm{zu}$ entbehren, weil bei dieser Procedur beide Pleurahöhlen eröffnet werden. Was die übrigen Einzelheiten in der Versuchsanordnung anbelangt, so will ich erwähnen, dass die Versuche, wenn künstliche Respiration angewendet werden sollte, mit einer Tracheotomie begannen: der lange Schenkel einer T-förmigen Canüle wurde in die Luftröhre eingebunden, während die kurzen Schenkel derselben durch Schläuche mit einem von Professor Thoma höchst sinnreich construirten Respirationsapparate in Verbindung standen. Ich überzeugte mich sebr bald davon, dass die künstliche Durchlüftung der unterbundenen Lunge resp. deren Gefässe die Versuchsthiere befähigte, die Infusion bedeutend längere Zeit hipdurch zu ertragen, - nur mittelst dieser künstlichen Respiration und ihrem rhythmischen Einund Ausathmen habe ich es vermocht, ihnen bis zu $950 \mathrm{Ccm}$. Infusionsmasse beizubringen. Und so wendete ich sie, ganz abgesehen davon, ob ich eine oder beide Pleurahöhlen gêouffnet hatte, schliesslich bei-allen meinen Versuchen an und glaube ihnen einen grossen Theil der günstigen Erfolge zuschreiben zu dürfen.

Nachdem die künstliche Respiration in geregeltem Gange war, machte ich auf der linken Seite eine ausgiebige Resection der 2. und 3 . oder 3 . und 4 . Rippe und unterband die Arteriae intercostales. Darau schritt ich zur Unterbindung der Lungengefässe. Der letzte Act der Versuchsanordnung bestand darin, dass ich eine Canüle in die Vena jugularis externa einband. Sie stand mittelst eines 3-4 Mm. dicken Gummischlauches mit einer graduirten Flasche, aus der die Infusionsflüssigkeit unter constantem Druck tropfenweise abfloss, in Verbindung. Zur Erwärmung der Flüssigkeit batte ich in den 1 Meter langen schlauch ein vielfach gewundenes Glasrohr eingeschaltet; das Glasrohr lag während der Dauer des Experiments in einem von einer Gasflamme warm erhaltenen Wasserbade. Durch Veränderung der Tropfenzahl war ich im Stande, jeder Zeit die Quantität des in die Vene überfliessenden Gemenges zu variiren resp. zu unterbrechen; der tropfenweisen Infusion, der Möglichkeit selbst diese nöthigenfalls unterbrechen zu können, wenn das Thier unruhig zu werdẹn begann, was in der 
Regel im Beginn des Experimentes geschah, der warmen Infusionsflüssigkeit habe ich es zu danken, dass keines von den vielen Versuchsthieren, die ich verwandte unter irgend welchen plötzlich auftretenden Erscheinungen zu Grunde ging, alle endeten unter den Erscheinungen eines tiefen Sopors. - Die Section wurde unmittelbar nach eingetretenem Tode vorgenommen; die Untersuchung der Lungen, stets mit einer Injection des unterbundenen Abschnittes der Gefässstämme vom Herzen aus eingeleitet, um den Nachweis zu führen, dass die vollständige Unterbindung eines bestimmien Gefässes auch wirklich ausgeführt war. Bevor ich zur mikroskopischen Untersuchung ging, goss ich die Lungen von der Trachea aus mit Leim voll, — das ausgedehnte Object macht eine eingehende Untersuchung der Gefässe möglich und wird in jeder Bcziehung zugänglicher.

Was die Resultate der Untersuchung anlangt, so waren sie bezüglich der Vertheilung des Zinnobers wesentlich dieselben. Da der Zinnober den mil Leim ausgedehnten Lungen die natürliche, hellrosenrothe Farbe gab, diese aber nicht-ausgesprochen genug ist, um ein Mehr oder Weniger beurtheilen zu können, so begnüge ich mich damit, zu constatiren, dass bei lange dauernder Infusion ( $8-9$ Stunden) beide Lungen gleichfarbig wàren, d. h. in beiden Lungen war Zinnober. Das Ligam. pleuro-oesophageum hatte mit Zinuober gefüllte Arterien und Venen neben Extravasaten zwischen den Pleurablättern, in denen Zinnoberkörnchen nachweisbar waren. Um die Arteria und Vena pulmonalis, den Bronchus in ihrem ganzen Verlauf übersichtlich vor sich zu haben, empfehle ich den Schnitt durch den linken unteren Lappen so zu führen, dass er von einer der Linea axillaris etwa entsprechenden Linie ausgehend, in der Richtung zum Hilus des Lappens geführt wird, - mit anderen Worten, man halbire den Lappen durch einen zum Hilus geführten Schnitt. Man ïberzeugt sich ohne weitere mikroskopische Untersuchung davon, dass die Stämme der Arteria und Vena pulmonalis, d. $h$. in Fällen, in denen beide unterbunden waren, mit zinnoberrothen Pfröpfen gefüllt, die je nach der Dauer des Experiments mehr oder weniger fest sind, - beide reichen bis zur Gefässligatur am Hilus. $\mathrm{Da}$ die zinnobergefärbten fibrinösen Gerinnsel ziemlich deutlich in den Gefässen zweiter Ordnung abbrechen, so hat es das Ansehen, wie wenn mit dem Verschluss der Stammgefässe die 
Circulation eine andere Richtung genommen habe. Auf der Schleimhaut der grossen Bronchien lag der Zinnober hie und da in beträchtlichen Mengen. Die Annahme, dass er aus den Gefässen der gesunden Lunge in den Bronchialbaum dieser und von da in die Bronchialverzweigungen und Gefässe der anderen Lunge übergegangen sei, lässt sich widerlegen durch die Versuche bei denen der Bronchus unterbunden war. Die Gefässe der Schleimhaut, also Aeste der Arteria pulmonalis, ebenso die des bronchialen Bindegewebes sind reichlich mit Zinnober gefüllt, - deutlicb erkennt man die ampullären Gefässe der Schleimhaut vermöge des zinnober-. haltigen Inhalts. Betrachtet man die Ränder der Lungen mit unterbundener Pulmonalarterie, so kann man auch ohne mikroskopische Untersuchung den Zinnober in ihnen nachweisen; Zinnoberkörnchen finden sich in den subpleuralen Randgefässen, ebenso in den Capillarbezirken der Alveolen. Diese waren häufig so bedeutend gefüllt, dass siè sich als continuirliche und prall gefüllte Capillarnetze über das Niveau der epithelialen Auskleidung erhoben. Auf dem Epithel der feineren Bronchien und der Alveolen lagen die Körnchen frei. Bei auffallendem Lichte (Lampenbeleuchtung) sah man das ganze Object von leuchtenden, feinsten Zinnoberkörnchen durchsetzt.

Die rechte Lunge, deren Arteria pulmonalis frei war, enthielt in den meisten Fällen beträchtlich mehr Zinnober, - bei Versuchen, in denen grosse Mengen ( $950 \mathrm{Ccm}$.) innerhalb längerer Zeit (9 Stunden) infundirt worden waren, fanden sich beide Lungen nabezu gleich, in einem Falle war die linke, d. b. unterbundene sogar mehr gefärbt.

Aus den zuletzt berichteten Infusionsversuchen geht als wichtige Thatsache mit Sicherheit hervor, dass bei Thieren, denen bei unterbundener Arteria pulmonalis Zinnober enthaltende Flüssigkeit (in beträchtlicher Menge, längere Zeit hindurch) in's Blut infundirt worden, dieser Farbstoff nicht nur in den Zweigen der Bronchialarterie, sondern auch in den Zweigen und in dem unterbundenen Stamm der Arteria pulmonalis, in den Capillaren der Alveolarwände, so wie in den Lungenvenen getroffen wird. Dieses Versuchsresultat lässt, meines Erachtens nach, nur eine Deutung zu, - nehmlich die, dass die Zufubr des zinnoberhaltigen Blutes durch Zweige der Arteriae bron- 
chiales, tracheo-oesophageae, oesophageae, mediastinales, pericardiacophrenicae, der Abfluss desselben durch die Vena azyoos und obere Hohlvene erfolgt sei. Man kann in den beiden letzteren den Zinnober direct nachweisen. Die grossen Mengen von Zinnober aber, welche in denjenigen Lungenabschnitlen gefunden werden, deren Pulmonalarterien unterbunden sind, ergeben einen weiteren Beweis dafür, dass die Blutströmung in diesen Abschnitten relativ sehr beträchtlich sei.

Ich darf diese Mittheilungen nicht schliessen, ohne auf die Uebereinstimmung des Resultats der anatomischen und experimentellen Untersuchungen hingewiesen zu haben. Beziiglich der Arteria pulmonalis ist in dem anatomischen Theil nachgewiesen worden, dass ihr Gefässgebiet keineswegs ein so abgeschlossenes ist, wie man dies hat gettend machen wollen, - dass vielmehr die einzelnen Zweige unter sich, wenn auch nur durch Capillaren, in ausgedehnter Verbindung stehen, dass insbesondere aber auch ausgiebige Beziehungen zwischen dem Gefässgebiet der Arteria pulmonalis und bronchialis bestehen, welche von um so grösserer Bedeutung sind, da die Zweige der letzteren unter sich durch arterielle Aeste in Verbindung steheo. Die oben berichteten Versuche können nur im Stande sein, die Bedeutung dieser anatomischen Einrichtung dahin zu illustriren, dass bei versehlossener Arteria pulmonalis den Lungen durch die Arteria bronchialis Blut zugeführt wird. Kommt die Blutzufuhr bei Unterbindung des Stammes der Arteria pulmonalis bereits zu Stande, so sind die Consequenzen, die sich für die Absperrung eines Astes derselben ergehen, ersichtlich.

Für die Frage, ob die Arteria pulmonalis Endarterie sei oder nicht, vermögen diese Versuche den Entscheid zu bringen. Mittelst der anatomischen Untersuchungen habe ich nicht vermocht präformirte Verbindungen nicht capillärer Art zwischen den Zweigen der Arteria pulmonalis nachzuweisen. Es ist aber in dieser und der früheren Arbeit betont worden, dass unter gegebenen Verhältnissen sich solche weitere Verbindungen herstellen können; berücksichtigt man die anatomische Configuration der Gefässbahn der Arteria pulmonalis, deren Beziebungen zu dem Gefässgebiet der Arteria bronchialis, sowie die Ergebnisse dieser experimentellen Untersuchungen, so wird man zugeben müssen, dass die Gelässverzweigung und der Bau der Capillarbahn ein sehr 
ausgiebiges Ueberströmen des Blutes von einem Gefässbezirk in den anderen ermöglicbt.

\section{Erklärung der Abbildungen. \\ Tafel XI-XII.}

Fig. 1. Verzweigung der Arteria pulmonalis an den scharfen Rändern einer Hundelunge bei intacten Pleuren. Der Gefässinjection ging eine Injection des Bronchialbaumes mit 0,25procentiger Silherlösung voraus, - die Venae pulmon. und Capillaren sind hiermit ausgeschaltet. Ausgedehnt ist die Lunge mit ungefärbtem Leim, der schliesslich in den Bronchialbaum injicirt wurde. Die Arteria pulmon. theilt sich in so viel Aeste als Infundibula vorhanden sind, - sie läuft auf den Kuppen derselben, - mit ihren Endästen greift sie in's subpleurale, interinfundibulare Bindegewebe oder sie tritt za den Nebentrichtern (b); künstliche Anastomosen bei a a a. (42fache Vergrösserung, -- stereoskop. Bild.)

Fig. 2. Die Lunge war nicht mit Silberlösung injicirt - die Art. pulmon. mit Berliner Blau injicirt, die Lunge ausgedehat. Verästelung der Arterie im subpleuralen Gewebe, - Uebergreifen der Aeste zu vachbarlichen Infundibeln.

Fig. 3. Mit 0,5procentiger Silberlösung injicirte Hundelunge, - mit Berliner Blau injicirte Art. pulm., - schliesslich ausgedehnt, - scharfer Lungenrand. Künstliche Anastomosen der Art. pulmonalis; ausgeschaltete Venen und Capillaren.

Fig. 4. Dieselbe Präparation der Lunge bis auf eine stärkere Concentration der Silberlösung $(1 \mathrm{pCt}$.). Mächtige künstliche Anastomosen; ausgeschaltete Venen, Capillaren und Arterienzweige.

Fig. 5. Verzweigung der Vena pulmonalis an den scharfen Rändern bei intacten Pleuren. Injection des Bronchialbaumes resp. Lunge mit 0,25procentiger Silberlösung - Ausschaltung der Art. pulm. und der Capillaren - mit ungefärbtem Leim ausgedehnte Lunge. Die Venen verlaufen in den Gewebseinsenkungen $d . h$. interalveolär oder interacinös. Stereoskopisches Bild. 42fache Vergrösserung. Ein Vergleieh von Fiğ. 5 mit Fig. 1 giebt charakteristische Unterschiede zwischen Arterien- und Venenverlauf.

Fig. 6. Ein Bronchus zweiter Ordnung aus der Lunge eines Neugebornen, auf dessen Oberfläche die spiraligen Windungen der Arteria bronchialis verlaufen; sie geben auffallend wenig Aeste überbaupt, namentlich aber dem Bronchins ab, - die mesiten verlaufen im peribronchialen Bindegewebe und umspinnen die Arter. pulmon., Lymphgefässe und Nerven. Ich habe hier eine Stelle gewählt, in welcher relativ starke Stämme zu den dem Bronchus angrenzenden Lungenlappen resp. Alveolen treten und sich an letzteren peripher ausbreiten. Die dem Bronchus unmittelbar anliegenden biau gezeichneten Gefüsse sind Zweige der Arter. pulmon. und zu grösseren Pulmonalvenen gehende Bronchialgefässe. Die Arter. bronchialis war von der Aorta ans gefültt. 
Fig. 7. Von der Aorta aus injicirte Lunge eines Neugebornen (4 Stunden gelebt), es ist ein der Lungenoberfläche entsprechend (parallel) geführter Schnitt, so dass man sich das Gefãssbild von Pleura gedeckt vorstellen muss; die Arteria und Venae pulmonales waren bei der Injection unterbunden. a $a$ a a Lungenläppchen. $b$ b b Interlobuläres Bindegewebe. c c Vom Lungenhilus bis an die Lungenoberfäche aufsteigende Pleuraarterien. Sie verbreiten sich theils im subpleuralen Bindegewebe, theils senken sie sich in die Läppchen ein und anastomosiren mit Stämmen der Arteria pulmonalis; die feineren Zweige breiten sich an den einzelnen Alveolen capillär aus. (Stereosk. Bild.)

Fig. 8. Der scharfe Lungenrand einer Hundelunge, - der Bronchialbaum war vor der Arterieninjection mit 0,5procentiger Silberlösung gefüllt, - die Arteria und Venae pulmon. am Stamm unterbunden, - dann folgte elne Injection der Aorta abdominalis nach aufwärts, - schliesslich wurde die Lunge mit ungefärbtem Leim ansgedehnt. Es versinnlicht das Bild die Anastomosen der Pleuraarterien (roth) mit den Art. pulmonales (schwarz), - es sind die in Fig. 7 erwähnten, in die Läppchen sich einsenkenden Pleuragefässe. Mit anderen Worten die Art. pulmonales waren von den Pleuragefássen aus gefüllt. Obschon beide mit einer und derselben Injectionsmasse gefüllt waren, so habe ich hier roth und schwarz gewählt, um die Einsenkung der Pleuragefässe in die Pulmonalarterien deutlich zu machen.

Flg. 9. Von der Ven. pulmon. aus injicirte Lunge eines 2wöchentlichen Kindes. a Schräg durchschnittener Bronchus mit dessen longitudinal verlaufenden Bronchialvenen $d d d d d$. Bei e verbinden sie sich mit einem Stamm, dessen Blut aus der Alveole $c$ datirt, die bedeutend ausgedehnt war, beide werden zu einem grossen Pulmonalvenensinus. b Arter. pulmonalis. An den angrenzenden Schnitten liess sich die Fortsetzung von e als starke, interlobulär verlaufende Vene constatiren. 